Canadian

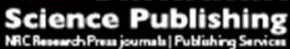

Canadian Geotechnical Journal Revue canadienne de géotechnique

\title{
Multi-Directional Force-Displacement Response of Underground Pipe in Sand
}

\begin{tabular}{|r|l|}
\hline Journal: & Canadian Geotechnical Journal \\
\hline Manuscript ID & cgj-2016-0059.R1 \\
\hline Manuscript Type: & Article \\
\hline Date Submitted by the Author: & $15-J u n-2016$ \\
\hline Complete List of Authors: & $\begin{array}{l}\text { Jung, Jai; Youngstown State University, Civil and Environmental } \\
\text { Engineering; } \\
\text { O'Rourke, Thomas; Cornell University, } \\
\text { Argyrou, Christina; Cornell University, Civil and Environmental Engineering }\end{array}$ \\
\hline Keyword: & $\begin{array}{l}\text { pipeline, Mohr-Coulomb, finite element analyses, soil-pipe interaction, } \\
\text { ground failure }\end{array}$ \\
\hline & \\
\hline
\end{tabular}


Multi-Directional Force-Displacement Response of Underground Pipe in Sand Jai K. Jung ${ }^{1}$, Thomas D. O’Rourke ${ }^{2}$, and C. Argyrou ${ }^{3}$

\begin{abstract}
A methodology is presented to evaluate multi-directional force-displacement relationships for soil-pipeline interaction analysis and design. Large-scale tests of soil reaction to pipe lateral and uplift movement in dry and partially saturated sand are used to validate plane strain, finite element (FE) soil and pipe continuum models. The FE models are then used to characterize force vs displacement performance for lateral, vertical upward, vertical downward, and oblique orientations of pipeline movement in soil. Using the force vs displacement relationships, the analytical results for pipeline response to strike-slip fault rupture are shown to compare favorably with the results of large-scale tests in which strike-slip fault movement was imposed on 250-mm and 400-mm-diameter high density polyethylene pipelines in partially saturated sand. Analytical results normalized with respect to maximum lateral force are provided on $360^{\circ}$ plots to predict maximum pipe loads for any movement direction. The resulting methodology and dimensionless plots are applicable for underground pipelines and conduits at any depth, subject to relative soil movement in any direction in dry/saturated and partially saturated medium to very dense sands.
\end{abstract}

KEY WORDS: pipeline, Mohr-Coulomb, soil-pipe interaction, earthquake, liquefaction, ground failure

1. Assistant Professor, Civil and Environmental Engineering, Youngstown State University, Youngstown, OH jai.k.jung@gmail.com, corresponding author

2 Professor, School of Civil and Environmental Engineering, Cornell University, Ithaca, NY tdo1@cornell.edu

3 Graduate Research Assistant, School of Civil and Environmental Engineering, Cornell University, Ithaca, NY ca353@cornell.edu 


\section{Introduction}

Of key importance in the response of underground pipelines to permanent ground deformation (PGD) is the reaction that develops as the pipe moves relative to the soil. It has long been recognized (e.g., Trautmann and O'Rourke 1985; ASCE 1984) that soil pressures associated with relative soil-pipe movement affect pipeline axial strain, curvature, and ovaling, as well as the relative slip and rotation at pipeline joints. Although industry guidelines cover force-displacement relationships for lateral displacement, vertical uplift, and settlement (e.g., ASCE 1984; Honegger et al. 2004), a comprehensive methodology supported by large-scale tests and continuum modeling of both the soil mass and embedded pipe is not available for predicting force vs pipe-soil relative displacement in all directions. Continuum models, however, are available that account for stress and deformation throughout soil adjacent to underground pipelines (e.g., Robert et al. 2016b; Vazouras et al. 2015; Jung et al. 2013a and 2013b; Xie at al. 2013; O'Rourke 2010), thus providing the framework for simulating in a realistic way pipeline performance under externally imposed ground deformation.

Figure 1 illustrates how soil-pipe force vs displacement relationships are used to analyze continuous pipeline response to externally imposed ground deformation. The pipe is modeled as a beam, often with nonlinear material and geometric properties, and soil-pipe interactions orthogonal and parallel to the pipeline longitudinal axis are modeled by linear, multi-linear, and nonlinear relationships. In the figure, bilinear relationships are shown for force vs longitudinal, lateral, and vertical upward and downward relative displacement between the pipe and soil. Recommendations for modeling pipeline response to ground deformation as depicted in the figure are readily available (e.g., ASCE, 1984; Honegger and Nyman 2004; O'Rourke et al. 2008). 
In this paper, a methodology is presented that uses large-scale soil-pipe interaction tests to characterize lateral and uplift force vs displacement relationships in combination with plane strain, finite element (FE) soil continuum models for soil-pipeline interaction. The finite element models are validated with respect to the large-scale soil-pipe interaction test results for lateral and upward relative displacement between pipe and soil, and then used to characterize pipe force vs displacement performance for vertical downward and oblique pipe movement in sand.

The analytical and experimental results are normalized with respect to maximum lateral force, and the normalized maximum forces are plotted on $360^{\circ}$ plots that provide maximum pipe loads for medium to very dense dry sand at various depth to diameter ratios. Guidance is provided to account for the effects of pipe diameter and suction in the characterization of soilpipe interaction. Pipeline response for strike-slip fault rupture using the force vs displacement relationships developed in this work are shown to be in favorable agreement with the results of three large-scale tests of strike-slip fault movement on high density polyethylene (HDPE) pipelines in sand.

\section{Soil Model}

The soil model employed in this work has been described in detail in previous publications (Jung et al. 2013a; Jung et al. 2013b) and shown to produce analytical results that are in excellent agreement with the results of large-scale tests of soil-pipe interaction for lateral and uplift movements. Soil behavior is represented by an elasto-plastic constitutive model with a Mohr-Coulomb (MC) yield surface and non-associated flow rule (Zienkiewicz et al. 1975). Soil strength is expressed by the peak plane strain friction angle, $\phi_{\mathrm{ps}-\mathrm{p}}$, cohesive intercept, $\mathrm{c}_{\mathrm{ps}}$, and peak dilation angle, $\psi_{\mathrm{p}}$. For partially saturated sand, $\phi_{\mathrm{ps}-\mathrm{p}}$ and $\mathrm{c}_{\mathrm{ps}}$ are total stress parameters. For dry sand, $\phi_{\text {ps-p }}^{\prime}$ and $c_{p s}^{\prime}$ are effective stress parameters. The secant soil modulus, $\mathrm{E}_{\alpha}$, is defined as 
strain compatible with the maximum pipe load, and a softening relationship developed by Anastasopoulos, et al. (2007) is used to simulate post peak soil shear strength.

The model adopted for this work focuses on the direct shear (DS) test. When used with appropriately designed DS equipment, sized to accommodate the range of particle diameters being tested, the DS test provides reliable measurements of key strength and dilatency parameters (e.g., Lings and Dietz 2004; O'Rourke 2010). A major benefit of the test is that it can be performed rapidly on large numbers of samples with different soil densities and moisture contents at a higher level of production and consistency than can be achieved with triaxial testing.

Only the salient features of the model are presented here under the subheadings of soil strength parameters, secant soil modulus, and strain softening behavior.

\section{Soil Strength Parameters}

Davis (1968) first derived the relationship between peak DS and plane strain shear, which is illustrated in Figure 2. Given that the DS test failure plane coincides with zero extension and that incremental stress and strain are coaxial (e.g., Bolton 1986; Lings and Dietz 2004), the peak shear stress, $\tau_{\mathrm{p}}$ measured in the DS test is oriented at the peak dilatency angle, $\psi_{\mathrm{p}}$, with respect to the vertical diameter of the Mohr circle at $\left(\sigma_{1}+\sigma_{3}\right) / 2$. The value of $\phi_{\mathrm{ps}-\mathrm{p}}$ is defined at the point of maximum obliquity with respect to the Mohr circle, and the geometric relationship between $\tau_{\mathrm{p}}$ and plane strain peak shear stress, $\tau_{\mathrm{ps}}$, is shown in the figure. Using this relationship Jung et al (2013b) derived for partially saturated sand a general equation for $\phi_{\mathrm{ps}-\mathrm{p}}$ as

$$
\sin \phi_{p s-p}=\frac{\tau_{p} / \sigma_{N}}{\left[1+\frac{\tau_{p}}{\sigma_{N}} \tan \left(\psi_{p}\right)_{p . s a t}+\frac{c_{p s} \cot \phi_{p s-p}}{\sigma_{N}}\right] \cos \left(\psi_{p}\right)_{p . s a t}}
$$


in which the parameters are expressed in total stress and $\tau_{\mathrm{p}} / \sigma_{\mathrm{N}}$ is the DS test ratio of peak shear to normal stress, $\psi_{\mathrm{p}}$ is the dilatency angle for partially saturated sand, and $\mathrm{c}_{\mathrm{ps}}$ is determined from DS test data as $c_{p s}=c / \cos \phi_{p s-p}$, as explained by Olson (2009) and O’Rourke (2010).

For dry sand under effective stress conditions with zero cohesion, $\tau_{\mathrm{p}}^{\prime} / \sigma_{\mathrm{N}}^{\prime}=\tan \phi_{\mathrm{ds}-\mathrm{p}}^{\prime}$, and Eqn (1) reduces to

$$
\sin \phi_{p s-p}^{\prime}=\frac{\tan \phi_{d s-p}^{\prime}}{\cos \psi_{p}+\sin \psi_{p} \tan \phi_{d s-p}^{\prime}}
$$

in which the parameters are expressed in terms of effective stress and $\phi^{\prime}{ }_{\text {ds-p }}$ is the peak effective stress friction angle.

Direct shear and large scale soil-pipe interaction tests were performed with dry and partially saturated RMS sand with water contents $=4 \%$ and dry Cornell University $(\mathrm{CU})$ filter sand. The grain size distributions of these glacio-fluvial sands are plotted in Fig.3. The RMS graded sand naturally drains in the large scale test basin to $\mathrm{w} \approx 4 \%$, which is equal to the residual water content (O'Rourke 2010). Thus, no matter how high the initial water content of the sand, it drained rapidly to about $4 \%$ in all tests. Soil properties are summarized in Table 1 . Additional information on angularity, minerology, and grain size characteristics are provided by Jung et al. (2013a and 2013b).

To make use of Eqns. 1 and 2, one needs to determine $\phi_{\text {ds-p }}^{\prime}, \phi_{\text {ds-p }}$, and $\psi_{\mathrm{p}}$ for dry and unsaturated conditions. Olson (2009) performed numerous DS tests to determine strength and dilatancy properties of both dry CU filter and dry and partially saturated RMS sands. Typical results for dry RMS sand are shown in Fig. 4 that present $\phi_{\text {ds-p }}^{\prime}$ and $\psi_{\mathrm{p}}$ as a function of dry unit weight, $\gamma_{\mathrm{d}}$, at a normal effective stress typical of many pipe burial depths $(10-20 \mathrm{kPa})$. 
Because dilatancy is diminished by increasing confining stress, it is necessary to characterize the relationship between $\psi_{\mathrm{p}}$ and either $\sigma^{\prime}{ }_{\mathrm{N}}$ or $\sigma_{\mathrm{N}}$. Olson (2009) provided such data. For example, $\psi_{\mathrm{p}}$ vs. $\sigma^{\prime}{ }_{\mathrm{N}}$ for dry RMS sand with $\gamma_{\mathrm{d}}=16.5 \mathrm{kN} / \mathrm{m}^{3}$ are normalized with respect to the reference stress $\sigma^{\prime}{ }_{N}=2.1 \mathrm{kPa}$ and plotted in Fig. 5. The nonlinear regression equation for $\psi_{\mathrm{p}} / \psi_{\mathrm{p} @ \sigma^{\prime} \mathrm{N} \text { Ref. }}$ is presented in the figure. The $\psi_{\mathrm{p}}$ from that datasets associated with Figs. 4 and 5 was used to obtain $\phi_{\text {ds-p }}^{\prime}$, using the relationship provided by Lings and Dietz (2004) as

$$
\tan \phi_{d s-p}^{\prime}=\frac{\sin \phi_{c r i t}^{\prime}+\sin \psi_{p}}{\cos \psi_{p}}
$$

in which $\phi_{\text {crit }}^{\prime}$ is critical friction angle. Olson (2009) developed plots similar to that in Fig. 5 for different $\gamma_{\mathrm{d}}$ for dry and partially saturated conditions, and reported $\phi^{\prime}$ crit for CU filter and RMS sand as $38.6^{\circ}$ and $40.8^{\circ}$, respectively.

\section{Secant Soil Modulus}

Implicit in using an elasto-plastic model to predict maximum pipe force is the selection of Young's modulus to be strain compatible with the level of stress in the soil at peak pipe load. Duncan and Chang (1970) showed that the stress-strain relationship for sand can be approximated by a rectangular hyperbola and an associated reduction factor, $\mathrm{R}_{\mathrm{f}}$, given by

$$
R_{f}=\frac{\left(\sigma_{1}^{\prime}-\sigma_{3}^{\prime}\right)_{f}}{\left(\sigma_{1}^{\prime}-\sigma_{3}^{\prime}\right)_{u l t}}
$$

in which $\left(\sigma^{\prime}{ }_{1}-\sigma^{\prime}{ }_{3}\right)_{\mathrm{f}}$ is the maximum stress difference and $\left(\sigma^{\prime}{ }_{1}-\sigma^{\prime}{ }_{3}\right)_{\mathrm{ult}}$ is the asymptotic value of the stress-strain curve when modeled as a rectangular hyperbola. The value of $R_{f}=0.9$ reported by Trautmann and O'Rourke (1985) was used in this study. Assuming that $\alpha$ is the fraction of the peak soil strength mobilized at maximum pipe force, $\mathrm{E}_{\alpha}$, is related to the initial tangent modulus, $\mathrm{E}_{\mathrm{i}}$, as 


$$
E_{\alpha}=\left(1-\alpha R_{f}\right) E_{i}
$$

From Eqn. 5, $\mathrm{E}_{\alpha}$ associated with some percentage, $\alpha$, of the maximum stress level can be calculated from $\mathrm{E}_{\mathrm{i}}$. As explained by Jung et al. (2013a and 2013 b), finite element simulations of pipe movement in sand were used to estimate the relationship between $E_{\alpha}$ and $E_{i}$ at peak pipe load. Since the maximum lateral pipe force is independent of E, FE results can be used without $a$ priori knowledge of $\mathrm{E}$ to estimate $\alpha$. Jung (2010) reported average values of $\alpha$ calculated from FE simulations of lateral, vertical upward, and vertical downward relative soil-pipe movement of $0.93,0.95$, and 0.80 , respectively. For each movement direction, $\alpha$ varied by approximately $\pm 5 \%$ depending on soil density. The reader should refer to Jung et al. (2013a and 2013 b) for additional details.

\section{$\underline{\text { Strain Softening Behavior }}$}

As explained by Jung et al. (2013a and 2013b), the model proposed by Anastasopoulos, et al. (2007) was used to diminish linearly both $\phi_{\text {pssp }}^{\prime}$ and $\psi_{\mathrm{p}}$ to residual values of $\phi_{\text {crit }}$ and 0 , respectively, from the plastic strain at $\phi_{\text {ps-p }}^{\prime}$ to the plastic strain at $\phi_{\text {crit, }}$ using the results of DS testing as

$$
\gamma_{f}^{p}=\frac{d_{x p}-d_{x y}}{H}+\frac{d_{x f}-d_{x p}}{d_{F E}}
$$

in which $\gamma_{\mathrm{f}}^{\mathrm{p}}=$ the plastic strain at $\phi_{\text {crit }}, \mathrm{H}=$ thickness of the DS specimen, $\mathrm{d}_{\mathrm{FE}}=\mathrm{FE}$ element size, and $d_{x y}, d_{x p}, d_{x f}$ are the DS test horizontal displacements at yield, peak strength, and $\phi_{c r i t}$ at which full softening occurs, respectively. The $\mathrm{d}_{\mathrm{FE}}$ in the refined part of the finite element mesh was taken as $20 d_{50}$, where $d_{50}$ is the median grain size. This thickness compares favorably with the shear band thickness as reported by several investigators as $8-20 \mathrm{~d}_{50}$ (e.g., Roscoe 1970; Vardoulakis and Graf 1985; Muhlhaus and Vardoulakis 1987; Huang, et al. 2002). A FORTRAN 
subroutine developed by Robert and Soga (2009) to apply the Anastasopoulos, et al. (2007) model in the MC model in ABAQUS was used in the 2D FE simulations.

\section{Validation of Modeling Process Relative to Large-Scale Test Results}

One of the first steps in developing the methodology was to perform FE simulations and compare the results with large-scale lateral and uplift soil-pipe interaction test results reported by Trautmann and O'Rourke (1985) and Olson (2009). Comparisons between representative analytical and large-scale test results are presented in Figs $6 a$ and $6 \mathrm{~b}$ and Figs. 6c and 6d for lateral and uplift pipe-soil interaction, respectively. The figure shows the dimensionless force, $\mathrm{F} /\left(\mathrm{H}_{\mathrm{c}} \bar{\gamma} \mathrm{DL}\right)$ vs dimensionless displacement, $\mathrm{Y} / \mathrm{D}$, for which $\mathrm{F}$ is the pipe-soil reaction force, $\bar{\gamma}=$ soil unit weight contributing to effective stress at the pipe centerline, $\mathrm{H}_{\mathrm{c}}=$ depth to pipe center, $\mathrm{D}$ $=$ outside pipe diameter, $\mathrm{L}=$ pipe length, and $\mathrm{Y}=$ relative soil-pipe displacement. Please note that the experimental and analytical test results reported for dry sand in this work pertain to effective stress conditions without suction generated in unsaturated sand. Effective stress conditions also apply to saturated sand in which the vertical effective stress equals the vertical total stress minus the porewater pressure. The conditions do not apply to liquefiable soils.

The large-scale test and analytical results compare favorably both for dry and partially saturated sand. Of particular interest is the simulation of strain softening behavior for lateral pipe movement in partially saturated sand with $\mathrm{H}_{\mathrm{c}} / \mathrm{D}=5.3$ (Fig. 6b) and uplift pipe movement in dry sand for $\mathrm{H}_{\mathrm{c}} / \mathrm{D}=4$ (Fig. 6c). A detailed comparison of test and analytical results with additional data and description of the modeling are provided by Jung et al. (2013a, 2013b).

\section{Lateral and Uplift Soil-Pipe Interaction}

Figure $7 \mathrm{a}$ presents plots of the maximum dimensionless lateral pipe force, $\mathrm{N}_{\mathrm{qH}}$, vs dimensionless depth, $H_{c} / D$, for dry medium, dense, and very dense sand for $H_{c} / D \leq 11$, based on 
both the large-scale test and analytical results provided by Jung et al. (2013b). Figure 7a also contains a plot of $\mathrm{N}_{\mathrm{qH}} \mathrm{vs} \mathrm{H}_{\mathrm{c}} / \mathrm{D}$ for partially saturated medium dense sand as reported by Jung et al. (2013b). Figure $7 b$ plots the maximum lateral pipe force throughout a broader range of $H_{c} / D$ $\leq 100$. Soil properties for medium, dense, and very dense sand are summarized in Table 2 . The dimensionless maximum pipe force is expressed as $\mathrm{F} /\left(\mathrm{H}_{\mathrm{c}} \bar{\gamma} \mathrm{DL}\right)$.

It should be noted that $\mathrm{N}_{\mathrm{qH}}$ in Fig. 7a is provided for $\mathrm{H}_{\mathrm{c}} / \mathrm{D} \geq 2$. Experimental evidence is sparse for pipelines buried with $0.5<\mathrm{H}_{\mathrm{c}} / \mathrm{D}<2$, and work performed on partially embedded submarine pipelines and shallow foundations (e.g., White et al. 2008) does not cover this range of very low $\mathrm{H}_{\mathrm{c}} / \mathrm{D}$. These considerations apply to vertical upward and vertical downward as well as lateral pipe displacement. It is recommended that, until further information is available, the dimensionless forces at $H_{c} / D=2$ be used as a generally conservative estimate for all orientations of pipe movement when $1<\mathrm{H}_{\mathrm{c}} / \mathrm{D}<2$.

In Fig. 7a the partially saturated sand results are strictly applicable only for the sand density, grain size characteristics, and water content used in this study. As discussed previously, the RMS sand drained rapidly to $\mathrm{w} \approx 4 \%$, irrespective of higher water content during placement and compaction in the large-scale tests. This condition is representative of water content sustained without infiltration, and is similar to pipeline burial in soil where overlying pavements block infiltration. Testing at this residual water content therefore has broader application to urban and suburban, paved environments for soils with similar grain size characteristics.

Figure 8 compares the dimensionless horizontal force vs displacement relationships for medium, dense, and very dense sand in this study with respect to similar relationships proposed by ASCE (1984) and ALA (2005) for sands with similar DS strengths. Although both sets of relationships incorporate data from Trautmann and O'Rourke (1985), the ASCE (1984) and ALA 
(2005) relationships underestimate $\mathrm{N}_{\mathrm{qH}}$ vs $\mathrm{H}_{\mathrm{c}} / \mathrm{D}$ for medium and dense sand compared with the results of this study. It should be recognized that Trautmann and O'Rourke (1985) used full-scale test data to select a model proposed by Ovesen (1964) to predict vertical anchor plate forces in sand, and it is the Ovesen model that results in underestimation of medium and dense sand forces. The analytical and experimental results in this study compare favorably with the original Trautmann and O'Rourke (1985) test results, and the results in Fig. 7 are recommended as the most reliable relationships for engineering practice.

Figure 9a presents plots of the peak dimensionless uplift pipe force, $\mathrm{N}_{\mathrm{qUV}}$, vs dimensionless depth, $H_{c} / D$, for dry medium and very dense sand for $H_{c} / D \leq 13$, based on the large-scale test and analytical results provided by Jung, et al. (2013a). The dimensionless pipe force here and elsewhere in this work is defined similarly to that for maximum dimensionless lateral pipe force in the previous section. Figure $9 \mathrm{~b}$ plots the maximum lateral pipe force throughout a broader range of $\mathrm{H}_{\mathrm{c}} / \mathrm{D} \leq 100$. The analytical model developed by Jung et al. (2013a) accounts for soil migration beneath the pipe through FE mesh adjustment coordinated with upward pipe displacement. For $\mathrm{H}_{\mathcal{c}} / \mathrm{D} \leq 5.5$, the model predicts decreasing uplift force after a peak value as upward movement of the pipe continues, which is consistent with experimental measurements. The FE results show that, at depths of $15-23 \mathrm{H}_{\mathrm{c}} / \mathrm{D}$, the soil movement around the pipe becomes symmetric (same movement pattern above and below the pipe). When a symmetric pattern of soil movement is established, the reaction force attains its maximum value, after which there is a very small force reduction at greater depth due to reduction in dilation angle with increased confining stress. .Similar to the comparison of analytical and experimental results for lateral soil-pipe interaction, a systematic comparison of FE model results with 
multiple full-scale test measurements of pipe response to uplift displacement in dry sand show excellent agreement both with respect to maximum force and force-displacement relationships.

The dimensionless uplift force vs displacement relationships in Fig. 9a are almost identical to those proposed by ASCE (1984) and ALA (2005). Because measurements reported by Trautmann et al. (1985) represent the core database used in this study as well as the empirical $\mathrm{N}_{\mathrm{qvu}}$ vs $\mathrm{H}_{\mathrm{c}} / \mathrm{D}$ plots presented by ASCE (1984) and ALA (2005), close agreement is expected between this work and the previous recommendations.

Because of the complexity involved with load transfer in loose sand, the methodology concentrates on medium to very dense sands only. Initially there is relatively low resistance to pipe movement in loose sand, with increasing resistance as sand in front of the pipe densifies with increasing pipe displacement. This type of interaction is sensitive to the initial sand density, and may be quite variable. If loose sands are encountered, the parameters for medium sands can be used for a conservative estimate of reaction forces.

\section{Finite Element Analysis for Downward Pipe Movement}

Because large-scale 2-D experimental results are not available for downward pipe movement in soil, FE analyses were used to simulate this mode of soil-pipe interaction. As explained in detail by Jung (2010) and O'Rourke et al. (2015), the FE modeling approach described above was applied to deep foundation bearing elements. The analytical results for the tip resistances of these deep foundations compare well with the tip resistances determined by widely used bearing capacity formulations (Kulhawy et al. 1983; Perkins and Madson 1996; Shiraishi 1990; Akbas and Kulhawy 2009b). The modeling is thus sufficiently robust for conventional tip bearing capacity assessment at variable depth, and is suitably structured for simulating downward pipe movement. 
Figure 10 shows a typical 2-D FE mesh for simulating vertical downward and oblique pipe movement in soil. Infinite elements (Zienkiewicz et al. 1983), were used to represent a semi-infinite soil medium. Inside the dashed lines, 8-node biquadratic, plane strain, quadrilateral, reduced integration elements (element type CPE8R) represent the soil surrounding the pipe. Outside the dashed line, five-node quadratic, plane strain, one-way infinite quadrilateral elements (element type CINPE5R) represent the semi-infinite soil medium and are attached to CPE8R elements. The soil medium and infinite boundary elements are $1.3 \mathrm{~m}$ to $11.7 \mathrm{~m}$ in width to eliminate boundary effects as the pipe nominal pipe diameter was varied from $102 \mathrm{~mm}$ to 900 mm. Approximately 1500 to 5000 elements were used, depending on $\mathrm{H}_{\mathrm{c}} / \mathrm{D}$ conditions. A refined mesh, approximately 4 pipe diameters wide, was centered on the simulated pipe.

The 12-mm-thick elements within the refined mesh were consistent in size with the shear band thickness observed during DS tests (Roscoe 1970; Vardoulakis and Graf 1985; Muhlhaus and Vardoulakis 1987; Huang et al. 2002). Sensitivity studies showed that the analytical results are not sensitive to thickness variations of 7 to $18 \mathrm{~mm}$, which is the range of shear band thickness observed by previous investigators.

Pipe movement was generated by imposing vertical downward and oblique displacements on all pipe nodes. Geostatic loading under $\mathrm{K}_{\mathrm{o}}=1$ conditions was applied to the soil and pipe at the beginning of the analysis. Soil stress cell measurements during large-scale tests at Cornell indicate that $\mathrm{K}_{\mathrm{o}} \approx 1$ is an appropriate representation of in situ soil stress conditions after pipe burial and soil compaction. Sensitivity studies for $\mathrm{K}_{\mathrm{o}}$ showed that the maximum force changed approximately $3 \%$ and the computational time using ABAQUS increased approximately 10 times when $\mathrm{K}_{\mathrm{o}}$ changed from 1 to 0.3 . To promote numerical stability, all simulations were performed with a nominal cohesion $\mathrm{c}_{\mathrm{ps}}^{\prime}=0.1-0.3 \mathrm{kPa}$, which was shown by sensitivity analyses 
to have a negligible effect on the analytical results. Separation and slip between soil and pipe were allowed in the analysis.

The pipe was modeled as a rigid cylinder, with a soil-pipe interface friction angle of 0.6

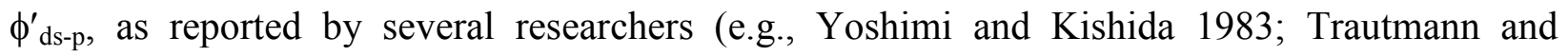
O'Rourke 1985; and Yimsiri et al. 2004). This interface friction angle is consistent with those measured for smooth polymer surfaces, reported by O'Rourke et al. (1990) on the basis of DS testing. Polymers with relatively hard, smooth surfaces are used frequently as pipe coatings. Since the soil-pipe interface is a surface of zero extension, the DS strength parameters, as illustrated in Fig. 1, are the appropriate ones for surface shear characterization. The DS test accounts properly for strength and dilatency associated with interface shear. Numerous researchers (e.g., O'Rourke et al. 1990) have shown that the interface friction angle, $\delta$, is proportional to $\phi^{\prime}{ }_{\mathrm{ds}-\mathrm{p}}$, thus accounting for increased $\delta$ with increased soil density that is reflected by increased $\phi^{\prime}$ ds-p.

\section{Analytical Results of Maximum Vertical Downward Force}

Methods recommended for the interpretation of deep and shallow foundation load tests (Hirany and Kulhawy 2002; Akbas and Kulhawy 2009a) were used to identify the maximum vertical force developed during each simulation. A typical load-settlement curve for an actual foundation test and FE simulation is shown in Fig. 11, for which. $\mathrm{L}_{1}$ is defined at the end of the initial linear region of the curve, and $\mathrm{L}_{2}$ is defined at the beginning of the final linear region. The vertical force associated with $\mathrm{L}_{2}$, referred to as the failure threshold, $\mathrm{Q}_{\mathrm{L} 2}$, by Akbas and Kulhawy (2009a), was used to determine the dimensionless maximum downward pipe force, $\mathrm{N}_{\mathrm{qVD}}$.

Table 2 summarizes the peak angle of shear resistance for the medium, dense, and very dense sand used in both the full-scale soil-pipe interaction tests and FE simulations. The strain 
compatible modulus, $\mathrm{E}_{\alpha}$, was calculated with $\alpha=0.8$, using Eq. 5, as discussed previously. The $\alpha$ parameter was selected after careful examination and analyses with numerous FE simulation results, as described by Jung (2010) and Jung, et al. (2013b).

Figure 12a presents the FE results for the dimensionless maximum vertical downward force $\left(\mathrm{N}_{\mathrm{qVD}}\right)$ related to medium, dense, and very dense sand for $\mathrm{H}_{\mathrm{c}} / \mathrm{D}=2-100$. The value of $\mathrm{N}_{\mathrm{qVD}}$ was calculated as the maximum measured force, $\mathrm{F}$, divided by $\bar{\gamma} \mathrm{H}_{\mathrm{c}} \mathrm{DL}$. It reaches a limiting value of 16,19 , and 24 for medium, dense, and very dense sand, respectively, between $H_{c} / D=8$ and 15. In the FE simulations the soil movement pattern above and below the pipe becomes symmetric at depths of $8-15 \mathrm{H}_{\mathcal{c}} / \mathrm{D}$. Similar trends are reported by Yimsiri et al. (2004) and Jung et al. (2013b) for lateral pipe movement, and Jung et al. (2013a) for upward pipe movement. Figure $12 \mathrm{~b}$ presents the FE results at an enlarged scale for $\mathrm{H}_{\mathrm{c}} / \mathrm{D}=2-11$. This plot facilitates the selection of $\mathrm{N}_{\mathrm{qVD}}$ for analysis and/or design purposes at lower, more commonly encountered $\mathrm{H}_{\mathrm{c}} / \mathrm{D}$. As $\mathrm{H}_{\mathrm{c}} / \mathrm{D}$ decreases, $\mathrm{N}_{\mathrm{qVD}}$ approaches a value analogous to the bearing capacity of a circular dished foundation with width $\mathrm{D}$ at the ground surface.

\section{Comparison between Pipe Peak Force and the Bearing Capacity for Deep Foundations}

Guidelines for the seismic design of oil and gas pipelines recommend that the maximum vertical force associated with downward pipe movement be estimated by conventional bearing capacity formulations (ASCE 1984; Honegger and Nyman 2004). It is of interest therefore to compare the maximum vertical pipe force from conventional bearing capacity theory with the peak pipe force given by the FE simulations.

The pipe bearing capacity was calculated according to widely used bearing capacity formulations (Vesić 1975; Kulhawy et al. 1983; Akbas and Kulhawy 2009b), which show that the maximum tip resistance of a deep foundation, $\mathrm{Q}_{\mathrm{tc}-\mathrm{bc}}$, can be estimated as 


$$
\mathrm{Q}_{\mathrm{tc}-\mathrm{bc}}=\left(q N_{q} \xi_{q r} \xi_{q s} \xi_{q d} \xi_{q i} \xi_{q g} \xi_{q t}\right) A_{t i p}
$$

This equation accounts comprehensively for the various factors affecting bearing capacity in which $q=$ vertical effective stress at $\mathrm{H}_{\mathfrak{c}}, N_{q}=$ bearing capacity factor, $\xi_{q r}=$ rigidity factor, $\xi_{q s}=$ shape factor, $\xi_{q d}=$ depth factor, $\xi_{q i}=$ load inclination factor, $\xi_{q g}=$ ground surface inclination factor, $\xi_{q t}=$ tilt of foundation factor, and $\mathrm{A}_{\text {tip }}=$ area of the base of the foundation. For this study, $\xi_{q i}, \xi_{q g}, \xi_{q t}$ factors are equal to 1.0 because all analyses were conducted for level ground conditions without any load inclination or eccentricity. For plane strain pipe loading, there is no adjustment in the shape factor, such that $\xi_{q s}=1$.

As shown by Kulhawy et al. (1983), bearing capacity for $\mathrm{H}_{\mathrm{c}} / \mathrm{D}>5$ is predominantly related to $\mathrm{N}_{\mathrm{q}}$, which is given by

$$
\mathrm{N}_{\mathrm{q}}=\mathrm{e}^{\pi \tan \phi^{\prime}} \tan ^{2}\left(45+\frac{\phi^{\prime}}{2}\right)
$$

The depth factor, $\xi_{q d}$, is relatively constant for the high $\mathrm{H}_{\mathrm{c}} / \mathrm{D}$ conditions under study at approximately $1.27,1.25$, and 1.23 for medium, dense, and very dense sand, respectively. The rigidity factor, $\xi_{q r}$, accounts for the compressibility of soil under bearing pressure. The procedure for computing $\xi_{q r}$ provided for sand by Akbas and Kulhawy (2009b) was followed in this work.

The bearing capacity vertical forces, $\mathrm{Q}_{\mathrm{tc}-\mathrm{bc}}$, were calculated with Eq. 7 for medium, dense, and very dense sand at $H_{c} / D=8,11,15,20$, and 30 . The peak force from the FE analyses associated with the $\mathrm{Q}_{\mathrm{L} 2}$ values previously described, $\mathrm{Q}_{\mathrm{tc}-\text { fea }}$, are plotted with respect to $\mathrm{Q}_{\mathrm{tc}-\mathrm{bc}}$ for $\mathrm{H}_{\mathrm{c}} / \mathrm{D}=8-30$ in Fig. 13. As shown in the figure, the failure threshold force for 2-D pipe 
settlement is consistently lower than the bearing capacity force for a deep foundation, with $\mathrm{Q}_{\mathrm{tc}-\mathrm{fea}}$ approximately $1 / 3$ of $Q_{\text {tc-bc. }}$

This reduction in vertical bearing resistance for an underground pipe relative to the conventional bearing capacity of a flat bearing surface at depth is corroborated by centrifuge test measurements reported by Xie et al. (2013), who report that the maximum vertical reaction force measured for underground pipe subjected to normal fault movement was between 1/3 and 1/10 the force derived from conventional bearing capacity formulations. White et al. (2008) show that the bearing capacity of a shallow foundation at the ground surface in sand is related to shape, with the bearing capacity of a conical circular foundation as low as $50 \%$ of the bearing capacity of a flat foundation with the same diameter.

It should be noted that downward movement of pipe is accompanied by soil migration into a zone in the soil mass directly above the pipe. Such movement is different than what occurs with deep foundation settlement, where the shaft above the bearing surface prevents soil migration into a similar zone directly above the base of the foundation. As a result, there is higher constraint on soil deformation that is accompanied by wider and deeper distribution of stresses for a deep foundation when compared to a pipe with similar bearing area.

Supplemental 2D FE analyses were performed on several vertically embedded walls, each with a circular base bearing surface equal in radius to that of a pipe at the same depth in the same soil. As described by Jung (2010), these vertical bearing elements were modeled with frictionless sides to facilitate direct comparison of the deep foundation tip and pipe vertical bearing capacities. The deep foundation tip bearing capacities were first checked with values obtained from conventional bearing capacity formulations, given by Eq. 7 above, and found to be in excellent agreement. The analyses show that the principal stresses and displacement vectors 
beneath the pipelines are concentrated in a narrow zone in contrast to the deep foundation elements that spread the principal stresses and displacement vectors more broadly and deeply.

\section{Analytical Results for Oblique Pipe Movement}

The semi-infinite soil-pipe FE models described above were used to investigate the relationship between maximum oblique forces and $H_{c} / D$. As illustrated in Fig. 10, the pipe was displaced an angle of $-45^{\circ}$ and $+45^{\circ}$ with respect to the horizontal for oblique downward and oblique upward FE analyses, respectively.

Figure 14a shows that the dimensionless maximum oblique downward force, $\mathrm{N}_{\mathrm{qOD}}$, reaches a maximum value at $H_{c} / D=16,18$, and 23 for medium, dense, and very dense sand, respectively, and then slightly decreases by about $2 \%$ from $H_{c} / D=15$ to 100 , reflecting the effects of increased confining stress at higher $\mathrm{H}_{\mathrm{c}} / \mathrm{D}$. Similarly, Figure 15a shows that $\mathrm{N}_{\mathrm{qOU}}$ reaches a maximum value at $H_{c} / D=20-30$ and then decreases by approximately $1 \%$ from $\mathrm{H}_{\mathrm{c}} / \mathrm{D}=30$ to 100 . Limiting values of $\mathrm{N}_{\mathrm{qOU}}$ are reached between $\mathrm{H}_{\mathrm{c}} / \mathrm{D}=20$ and 30 at 15,17 , and 21 for medium, dense, and very dense sand, respectively.

Figures $14 \mathrm{~b}$ and $15 \mathrm{~b}$ present the $\mathrm{FE}$ results at an enlarged scale for $\mathrm{H}_{\mathrm{c}} / \mathrm{D}=2-11$. These plots facilitate the selection of $\mathrm{N}_{\mathrm{qOD}}$ and $\mathrm{N}_{\mathrm{qOU}}$ for analysis and/or design purposes at lower, more commonly encountered $\mathrm{H}_{\mathrm{c}} / \mathrm{D}$.

\section{Relationship between Force and Pipe Movement Direction}

Equations for estimating the maximum oblique pipe force from known horizontal and vertical forces were proposed by Nyman (1984) with reference to Meyerhof (1973), and adopted in a modified format in guidelines for pipeline design (ASCE 1984). In this work, the equations proposed by Nyman (1984) are applied in accordance with Fig. 16, which shows how the pipe movement direction is defined relative to the top and invert of the pipe for oblique upward and 
oblique downward movement, respectively. The equations for the maximum dimensionless pipe force are

$$
N_{q O U}=i_{U} N_{q V U}
$$

and

$$
N_{q O D}=i_{D} N_{q V D}
$$

in which

$$
\begin{gathered}
i_{U}=1+\left(\frac{0.25 \beta_{u p}}{90^{\circ}-0.75 \beta_{u p}}\right)\left(\frac{N_{q H}}{N_{q V U}}-1\right) \\
i_{D}=1+\left(\frac{0.25 \beta_{\text {down }}}{90^{\circ}-0.75 \beta_{\text {down }}}\right)\left(\frac{N_{q H}}{N_{q V D}}-1\right)
\end{gathered}
$$

In the equations, $\mathrm{N}_{\mathrm{qOU}}, \mathrm{N}_{\mathrm{qVU}}, \mathrm{N}_{\mathrm{qOD}}$, and $\mathrm{N}_{\mathrm{qVD}}$ are the maximum dimensionless oblique upward, vertical upward, oblique downward, and vertical downward force, respectively, and $i_{U}$ and $i_{D}$ are inclination factors for oblique upward and oblique downward pipe force, respectively. The angle, $\beta_{\text {up }}$, is the angle defining the direction of movement with respect to vertical at the pipe crown, as illustrated in Fig. 16. The angle, $\beta_{\text {down }}$, is the angle defining the direction of movement with respect to vertical at the pipe invert, as illustrated in Fig. 16. The inclination factors in Eqs. 10 and 11 are both defined relative to $\mathrm{N}_{\mathrm{qH}}$, which is the maximum dimensionless lateral pipe force.

Using $\mathrm{N}_{\mathrm{qVU}}$ and $\mathrm{N}_{\mathrm{qH}}$ for various depths and sand unit weights as reported by Jung et al. (2013a and 2013b) and presented in part in Table 2, as well as the $\mathrm{N}_{\mathrm{qVD}}$ values presented in this study, $\mathrm{N}_{\mathrm{qOU}}$ and $\mathrm{N}_{\mathrm{qOD}}$ were estimated by Eqs. $9-12$ and identified as $\mathrm{N}_{\mathrm{qOU}-\mathrm{s}}$ and $\mathrm{N}_{\mathrm{qOD}-\mathrm{s}}$, respectively. For medium, dense, and very dense dry sand, the maximum dimensionless oblique upward and downward pipe forces were determined from FE analyses for $\beta_{\text {up }}=22.5^{\circ}$ and $45^{\circ}$, and $\beta_{\text {down }}=22.5^{\circ}$ and $45^{\circ}$, respectively. These FE results for maximum dimensionless oblique 
upward and downward forces are identified as $\mathrm{N}_{\mathrm{qOU}-\mathrm{FE}}$ and $\mathrm{N}_{\mathrm{qOD}-\mathrm{FE}}$, respectively. Figures $17 \mathrm{a}$ and b are plots of $\mathrm{N}_{\mathrm{qOD}-\mathrm{FE}}$ Vs $\mathrm{N}_{\mathrm{qOD}-\mathrm{s}}$ and $\mathrm{N}_{\mathrm{qOU}-\mathrm{FE}} \mathrm{Vs} \mathrm{N}_{\mathrm{qOU}-\mathrm{s}}$ for $\beta=45^{\circ}$, respectively, and Figs. 13c and $\mathrm{d}$ are plots of $\mathrm{N}_{\mathrm{qOD}-\mathrm{FE}}$ Vs $\mathrm{N}_{\mathrm{qOD}-\mathrm{s}}$ and $\mathrm{N}_{\mathrm{qOU}-\mathrm{FE}}$ Vs $\mathrm{N}_{\mathrm{qOU}-\mathrm{s}}$ for $\beta=22.5^{\circ}$, respectively. All figures show excellent agreement between the dimensionless oblique forces from FE analyses and those estimated from the simplified approach with $\mathrm{r}^{2}=0.99$. The average difference between $\mathrm{N}_{\mathrm{qOD}-\mathrm{FE}}$ and $\mathrm{N}_{\mathrm{qOD}-\mathrm{s}}$ is $3 \%$ and the average difference between $\mathrm{N}_{\mathrm{qOU}-\mathrm{FE}}$ and $\mathrm{N}_{\mathrm{qOU}-\mathrm{s}}$ is $5 \%$, indicating that Eqs $9-12$ are effective for estimating oblique forces.

The FE maximum dimensionless force for lateral, vertical upward/downward, and oblique orientations are combined in a series of graphs to visualize the variation in maximum pipe force for any movement direction under plane strain conditions. The analytical results are normalized with respect to maximum dimensionless horizontal force, $\mathrm{N}_{\mathrm{qH}}$, reported by Jung et al. (2013b). The normalized maximum forces are provided on $360^{\circ}$ plots that can be used to estimate maximum pipe loads for medium to very dense dry sand at various $\mathrm{H}_{\mathfrak{c}} / \mathrm{D}$.

Figures 18 and 19 show the ratio of dimensionless maximum force at any orientation and dimensionless maximum horizontal force, $\mathrm{N}_{\mathrm{q}} / \mathrm{N}_{\mathrm{qH}}$, plotted for $\mathrm{H}_{\mathrm{c}} / \mathrm{D}=3.5-100$. Please note that the dimensionless force ratio, $\mathrm{N}_{\mathrm{q}} / \mathrm{N}_{\mathrm{qH}}$, has a unique value for a given $\mathrm{H}_{\mathrm{c}} / \mathrm{D}$ and direction of pipe movement, which does not depend on sand density and associated friction angles. At low values of $\mathrm{H}_{\mathrm{c}} / \mathrm{D}$, the plots are asymmetric about the horizontal axis, with low $\mathrm{N}_{\mathrm{q}} / \mathrm{N}_{\mathrm{qH}}$ for upward pipe movement and high $\mathrm{N}_{\mathrm{q}} / \mathrm{N}_{\mathrm{qH}}$ associated with downward pipe movement. At $\mathrm{H}_{\mathrm{c}} / \mathrm{D}=30$, the graph is nearly symmetric, and at $\mathrm{H}_{\mathrm{c}} / \mathrm{D}=100$, the graph is a circle. Circular symmetry is achieved at large $\mathrm{H}_{\mathrm{c}} / \mathrm{D}$ where the maximum force is identical in all directions for isotropic soil conditions.

\section{Pipe Diameter Effect on Soil-Pipe Interaction Forces}


Various FE analyses were performed with various pipe diameters to investigate the effects of increasing diameter on soil-pipe interaction at constant $\mathrm{H}_{\mathrm{c}} / \mathrm{D}$. Figures $20 \mathrm{a}$ and $\mathrm{b}$ are plots of $\mathrm{N}_{\mathrm{qH}}$ and $\mathrm{N}_{\mathrm{qVD}}$, respectively, for various pipe diameters. Lateral and vertical downward soil reaction to pipe movement is related to the depth-dependent characteristics of soil strength as described by Jung et al. (2013b). For a given $\mathrm{H}_{\mathrm{c}} / \mathrm{D}$, increasing $\mathrm{D}$ results in increasing confining stress with depth, which decreases the dilation angle, $\psi_{\mathrm{p}}$, and peak angle of shear resistance, $\phi_{\text {ps- }}^{\prime}$ p. Smaller $\mathrm{N}_{\mathrm{qH}}$ and $\mathrm{N}_{\mathrm{qVD}}$ are mobilized by larger diameter pipe at relatively low values of $\mathrm{H}_{\mathrm{c}} / \mathrm{D}$. This trend is more pronounced for large $\psi_{\mathrm{p}}$ and $\phi_{\mathrm{ps}-\mathrm{p}}^{\prime}$, which results in a more rapid reduction in strength with depth. For medium sand with little dilatancy, the effects of pipe size are small to negligible.

The effect of pipe size is more pronounced for vertical downward movement compared with lateral displacement of the pipe. There was no significant effect of pipe size for vertical upward movement in the analytical results. For lateral displacement, the decrease in $\mathrm{N}_{\mathrm{qH}}$ with increasing diameter is relatively low and confined mostly to $\mathrm{H}_{\mathrm{c}} / \mathrm{D} \leq 6$. For vertical downward movement, the effect of diameter on diminishing $\mathrm{N}_{\mathrm{qVD}}$ are significant, especially for $\mathrm{H}_{\mathrm{c}} / \mathrm{D} \leq 8$. Additional large-scale testing is recommended to help quantify this condition as well as to provide guidance for treating pipelines greater than $900 \mathrm{~mm}$ in diameter.

\section{Soil Suction Effects}

Pipelines are most often constructed in unsaturated soils, where a meniscus forms between soil particles, creating suction that binds the particles together. As a result, the unsaturated soil becomes stiffer and stronger than its dry or fully saturated equivalent. Soil suction may increase soil-pipe interaction forces in partially saturated sands, and should be 
considered in analysis and design, especially for fine sand and granular soil with significant siltsized particles ( $>10 \%$ by weight).

Figure 7a contains a plot of $\mathrm{N}_{\mathrm{qH}} \mathrm{vs} \mathrm{H}_{\mathrm{c}} / \mathrm{D}$ for partially saturated sand as reported by Jung et al. (2013b). The dry and partially saturated experimental sands used to develop Fig. 7a are similar with respect to mineral content, grain size distribution, and angularity, and were involved in large-scale soli-pipe interaction tests at the same confining stresses and similar dry unit weights. Thus, a direct comparison of $\mathrm{N}_{\mathrm{qH}}$ for partially saturated vs dry medium sand in Fig. 7a provides an estimate of the increase in $\mathrm{N}_{\mathrm{qH}}$ associated with suction. There is an increase of 10 $20 \%$ in $\mathrm{N}_{\mathrm{qH}}$ for partially saturated medium dense sand relative to dry medium dense sand. The partially saturated sand results shown in Fig. 7a are strictly applicable only for the sand density, grain size characteristics, and water content used in this study, although as discussed previously, the results at residual water content have broader implications for pipeline burial in which urban and suburban settings where overlying pavements block water infiltration.

To expand on applications for unsaturated sands, reference is made to the work of Robert et al. (2016a) in which a constitutive model for suction effects was implemented in FE simulations of soil-pipe interaction and validated by comparison with large-scale test results conducted in two different sands at two different laboratories under both dry and partially saturated conditions. Unsaturated soil behavior was simulated by the unsaturated version of NorSand, which is a generalised constitutive model for sand based on critical state theory (Jefferies, 1993). The unsaturated version of Nor-Sand includes the effect of increasing apparent tensile strength due to the water meniscus at the particle contacts (cohesion enhancement) as well as enhanced dilation (dilation enhancement). These two hardening effects, as well as the classical 
plastic hardening effect, results in a triple-hardening model. The reader should refer to Robert et al. (2016a and 2016 b) for additional details.

Figure 21 is adapted from data presented by Robert et al. (2016a) in which grain size distribution zones are presented within the limits of ASTM Class II backfill (ASTM D 2321-11 2011). Please note that the methodology proposed in this paper does not include a model that accounts explicitly for suction, but rather incorporates the findings of the modeling by Robert et al. (2016a) through reference to Fig. 21. It provides guidance on the basis of general trends delineated by Robert at al. (2016a) that are shown in the figure.

The zones in the figure cover grain size distributions associated with negligible, low, moderate, and high potential for suction increasing lateral soil-pipe reaction forces for partially saturated sands relative to dry sands. For grain size distributions falling within the zones for moderate and high suction, lateral soil-pipe reaction forces for unsaturated sand may be as large as two and three times, respectively, of lateral soil-pipe forces for dry sand. Figure 21 is also useful for identifying partially saturated soil and backfill for which little to no increase in lateral pipe load is anticipated relative to dry sand conditions. Partially saturated soil with a grain size distribution within or to the left of the negligible suction potential zone will generate soil reaction forces equivalent to those in dry sand.

In the model developed by Robert et al. (2016a), lateral soil-pipe forces are provided for a degree of saturation corresponding to maximum suction. Hence, the model provides an upper bound estimate of suction-related lateral pipe force. The suction that develops in the field, however, may vary spatially and temporally in response to infiltration and migration of water in the unsaturated, or vadose, zone. A pipeline may be buried in partially saturated sand with suction considerably below the maximum levels reflected in the model. Given the variable and 
complex nature of suction, the principal value of Fig. 21 is to check the potential for suctionrelated effects. If suction-enhanced soil reaction is possible and represents a loading with potentially severe consequences for the pipeline, trench backfill using soils with negligible suction can be used or adjustments in pipe strength and wall thickness can be made to offset the effects of suction-enhanced forces on the pipelines.

\section{Force vs Displacement Relationships}

The transverse force vs displacement relationships for pipelines in dry sand, which are subject to different interaction modes (im) involving different directions of pipe movement, can be represented by a 1) rectangular hyperbola for the nonlinear characterization of force vs. displacement or 2) simple bilinear relationship between force and displacement (e.g., Trautmann and O’Rourke 1985; ASCE 1984; Trautmann et al. 1985). In dimensionless terms the general form of the hyperbolic equation is

$$
F^{\prime}{ }_{i m}=\frac{Y_{i m}^{\prime}}{A+B Y_{i m}^{\prime}}
$$

in which $\mathrm{F}^{\prime}{ }_{\mathrm{im}}=\left(\mathrm{F}_{\mathrm{im}} / \bar{\gamma} \mathrm{H}_{\mathrm{c}} \mathrm{DL}\right) / \mathrm{N}_{\mathrm{qim}} ; \mathrm{Y}^{\prime}{ }_{\mathrm{im}}=\left(\mathrm{Y}_{\mathrm{im}} / \mathrm{D}\right) /\left(\mathrm{Y}_{\mathrm{mim}} / \mathrm{D}\right) ; \mathrm{Y}_{\mathrm{mim}}$ is $\mathrm{Y}_{\mathrm{im}}$ corresponding to maximum force, $\mathrm{F}_{\text {mim }} ; \mathrm{F}_{\mathrm{im}}$ and $\mathrm{Y}_{\mathrm{im}}$ are force and displacement, respectively; and $\mathrm{N}_{\mathrm{qim}}$ is the dimensionless maximum force. The interaction modes (im) covered in this work include horizontal (H), vertical upward (VU), vertical downward (VD), oblique upward (OU), and oblique downward (OD) directions of pipe movement.

Figure 22a shows both the hyperbolic and bi-linear dimensionless force vs dimensionless displacement relationships. As recommended by Trautmann and O'Rourke (1985) for a bilinear relationship, the initial elastic portion has a slope of $\mathrm{K}_{70 \mathrm{im}}$ that intersects the hyperbolic relationship at $70 \%$ maximum force, such that approximately equal areas exist between $\mathrm{K}_{70 \mathrm{im}}$ and the hyperbolic trend below and above the $70 \%$ peak force value.

In Fig. 22a the slope $\mathrm{K}_{70 \mathrm{im}}=\mathrm{F}_{\mathrm{im}}^{\prime} / \mathrm{bY}_{\mathrm{im}}^{\prime}$. Recognizing that $\mathrm{F}_{\mathrm{im}}$ corresponds to the 
maximum dimensionless force, $\mathrm{N}_{\mathrm{qim}}$, and $\mathrm{Y}^{\prime}{ }_{\mathrm{im}}$ corresponds to the displacement at maximum force, $\mathrm{Y}_{\text {mim }}$, results in the following expression

$$
K_{70 i m}=\frac{N_{q i m} \bar{\gamma} H_{c} D L}{b Y_{\text {mim }}}=\frac{N_{q i m} \bar{\gamma} D L}{b\left(Y_{\text {mim }} / H_{c}\right)}
$$

Following the experimental findings of Trautmann and O'Rourke (1985) and the analytical results of Jung (2010) and Jung et al. (2013a), Table 3 summarizes the values of A, B, b, and $\mathrm{Y}_{\text {mim }}$ expressed in terms of $\mathrm{H}_{\mathrm{c}}$ that pertain to various interaction modes, including vertical upward, oblique upward, horizontal, oblique downward, and vertical downward relative soil-pipe displacement. Jung (2010) plotted the dimensionless results of FE simulations performed for each of the interaction modes listed in Table 3 for various soil densities, diameters, and $H_{c} / D$ and fit the dimensionless data for each mode with a rectangular hyperbola. The coefficient of determination, $\mathrm{r}^{2}$, for each interaction mode regression equation is listed in Table 3 . The parameters summarized in Table 3 can be used in Eqs. 13and 14 to estimate bi-linear and nonlinear relationships between soil-pipe interaction force and relative displacement.

Consider, for example, the horizontal, or lateral, displacement mode (im $=\mathrm{H})$. Largescale test and analytical data for lateral soil-pipe interaction in dry sand reported by Jung, et al (2013b) are plotted in Figure 22b, showing the maximum lateral dimensionless force, $\mathrm{N}_{\mathrm{qH}}$, vs. the dimensionless displacement associated with maximum force, $\mathrm{Y}_{\mathrm{mH}}$. The figure also shows the average hyperbolic relationship given by Eq. 13 and Table 3 that best fits the data with $r^{2}=0.98$ as

$$
F_{H}^{\prime}=\frac{Y_{H}^{\prime}}{0.20+0.82 Y^{\prime}{ }_{H}}
$$

Trautmann and O'Rourke (1985) and Jung (2010) showed that $\mathrm{Y}_{\mathrm{mim}} / \mathrm{H}_{\mathrm{c}}$ is relatively constant as $\mathrm{H}_{\mathrm{c}} / \mathrm{D}$ increases for dry medium, dense, and very dense sand. The appropriate values of $\mathrm{Y}_{\text {mim }}$ expressed in terms of $\mathrm{H}_{\mathrm{c}}$ for each interaction mode and sand density category are summarized in Table 3. 
The bilinear relationship in Fig. 22b that best fits the data for dry sand follows an initial linear equation given by $\mathrm{F}_{\mathrm{H}}^{\prime}=2 \mathrm{Y}_{\mathrm{H}}^{\prime}$. Large-scale tests indicate that $\mathrm{K}_{70 \mathrm{H}}$ for partially saturated RMS sand is about two to three times as high as that for dry sand (Jung, 2010). Increasing the slope, such that $\mathrm{F}_{\mathrm{H}}^{\prime}=4-6 \mathrm{Y}_{\mathrm{H}}^{\prime}$, provides an estimate of the increased stiffness that accounts for suction effects.

When using the hyperbolic relationship, Eq. 13 is transformed to site specific conditions by multiplying $\mathrm{F}^{\prime}$ im by the appropriate $\mathrm{N}_{\mathrm{qH}}$ for dry or partially saturated conditions and then converting the resulting dimensionless force through multiplication with the product of $\mathrm{H}_{\mathrm{c}} \bar{\gamma} \mathrm{D}$ to obtain force per unit distance. For dry sand, $\mathrm{Y}^{\prime}{ }_{\mathrm{im}}$ is multiplied by $\mathrm{Y}_{\mathrm{mim}}$ as estimated from the values in Table 3 . For partially saturated sand, values of $1 / 2$ to $1 / 3 \mathrm{Y}_{\text {mim }}$ will provide a suitable estimate of increased stiffness.

\section{Comparison of Analytical and Test Results}

To test the methodology, simulations of soil-pipeline interaction under strike slip fault displacements were performed with the FE computer program ABAQUS (2014), using the pipe force vs. displacement relationships presented in this paper. A detailed description of the testing and numerical simulation is provided by O'Rourke et al. (2008 and 2015), and only the salient features are given here. Figure 23 shows the split-basin testing facility at Cornell University that utilizes as much as 90 metric tons of partially saturated sand per test with maximum 1.2-1.8 m of strike-slip displacement. Large-scale tests were conducted with this facility on HDPE pipelines on nominal 250-mm and 400-mm-diameters HDPE pipelines subjected to $1.22 \mathrm{~m}$ of strike-slip displacement at a crossing angle of $65^{\circ}$ with respect to vertical plane of ground rupture. The depth of cover was $900 \mathrm{~mm}$ to top of pipe in all tests. The pipeline was instrumented with over 100 strain gages, many with the capability of measuring strains as high as $20 \%$. All tests were 
conducted with partially saturated RMS sand, with suction of 4-5 kPa measured by tensiometers (O'Rourke et al. 2008) and strength and stiffness consistent with dense dry sand.

The FE analyses were performed in accordance with the methodology proposed in this work. Strain rate and temperature effects of the HDPE were taken into account with stress vs strain data from direct tension tests in an environmental chamber under constant temperature and strain rates (Merry and Bray 1997). The FE model was composed of 43 beam elements, 168 spring-slider elements, and 84 gap elements to represent lateral and axial force vs displacement relationships (O'Rourke et al. 2015). The analyses were performed with a bilinear lateral force vs displacement relationship obtained from Eq. 14, Fig. 7 and the bilinear parameters in Table 3. Simulations of soil-pipeline interaction under strike-slip and normal fault displacements were performed using FE analyses accounting for coupled normal and frictional forces so that the longitudinal frictional force along the pipeline is related to the soil-pipe reaction force normal to the longitudinal axis of the pipe, $p_{N}$, by a simple Coulomb friction law, $p_{N} \tan \delta$, where $\delta$ is the angle of friction at the soil-pipe interface as described by O'Rourke et al. (2015).

The dry sand FE simulations were performed with $\mathrm{N}_{\mathrm{qH}}$ for medium sand from Fig. 7a, and the partially saturated FE simulations were performed with $\mathrm{N}_{\mathrm{qH}}$ for partially saturated sand in the same figure. For the bilinear force vs. displacement relationships, the displacement at which the full force is activated, was equal to $0.037 \mathrm{H}_{\mathrm{c}} \mathrm{b}=0.037(1 \mathrm{~m})(0.5)=0.0185 \mathrm{~m}$ for medium dry sand, using the $Y_{\text {mim }}$ provided in Table 3. For the partially saturated sand the displacement was halved, thus doubling the stiffness as recommended previously.

Figures 24a and $\mathrm{b}$ compare the numerical and measured axial and bending strains for large-scale tests on a 250-mm and two 400-mm HDPE pipelines, respectively, at $1.22 \mathrm{~m}$ of strike-slip displacement. Axial strains are calculated as the average pipe crown and invert strains, 
and bending strains are determined as one half the difference between the springline strains. Insert diagrams in the figure show the crown, springline, and invert locations.

Figure 24a shows that the numerical and experimental bending strains for the $250-\mathrm{mm}$ diameter HDPE pipeline follow similar distributions. The maximum experimental bending strains are best matched by analytical results that account for suction. The numerical and experimental axial strains likewise follow similar distributions, with the maximum FE axial strain for dry and partially saturated sand between $1 \%$ lower and $10 \%$ higher, respectively, than the measured axial strain.

The FE simulations for the 250-mm HDPE pipeline, the results for which are shown in Fig. 24a, were also run with a hyperbolic relationship for partially saturated sand, with results virtually the same as shown for a bilinear lateral force vs displacement relationship. In pipeline fault crossing simulations the maximum lateral force, which is the same for bi-linear and hyperbolic models, is mobilized at a very small level of abrupt soil movement so that the results are relatively insensitive to the type of force vs displacement relationship used. For problems involving spatially continuous soil movement associated with liquefaction-induced subsidence or movement distributed across a fault zone, significant differences may occur in the analytical results arising from bi-linear and hyperbolic force vs displacement relationships.

Figure $24 \mathrm{~b}$ presents the measured and simulated strains for two $400-\mathrm{mm}$-diameter HDPE pipelines. Several tactile pressure sensors were installed south of the fault rupture, as shown in the figure, which resulted in a zone where very low shear stress was transmitted to the sensor and pipe. Reduced longitudinal friction in this zone was modeled by $\tan \delta=0.1$. With nearly half the pipeline south of the ground rupture isolated from the full effects of soil shear forces, additional load was conveyed asymmetrically to each end of the test pipelines, with higher axial strains at 
the south end. This asymmetric distribution of axial strain is well represented by the FE results. Measured strains at the center of the pipeline appear to be influenced by the adjacent tactile pressure sensor, but otherwise are mostly within $10 \%$ of the analytical values.

The favorable comparisons of analytical and experimental results for strike-slip fault displacements, based on the 2-D lateral soil-pipe interaction tests and FE simulations presented in this work, are corroborated by additional favorable comparisons between analytical and centrifuge test results for vertical normal fault displacement effects on HDPE pipe, as explained by O'Rourke et al. (2015). The FE analyses for normal faulting effects on HDPE pipe used the maximum uplift and downward soil reaction forces and force vs displacement relationships described in this work. It appears, therefore, that the maximum forces and force vs displacement relationships based on 2-D conditions account reasonably well for soil-pipe interaction, provided that 3-D pipe deformation (cross-sectional pipe deformation) is relatively small. It should be recognized that soil deformation under plane strain 2-D conditions mobilizes higher strength and stiffness that can be attained under 3-D conditions so that 2-D reaction forces are more conservative than their 3-D counterparts.

\section{Application of Methodology}

Figure 25 presents a flow chart for estimating the maximum soil-pipe reaction force. First, the pipe and soil parameters are determined, including pipe depth and outside diameter direction of pipe movement, effective sand unit weight, and sand density. Next, the effects of pipe size are estimated with respect to Fig. 20. Finally, the effects of suction are evaluated with respect to Fig. 21. To illustrate the application of the methodology, two examples are provided as follows: 
Example 1: A nominal 300-mm ductile iron pipe with $335-\mathrm{mm}$ outer diameter and depth to centerline of $\mathrm{H}_{\mathrm{c}}=1.5 \mathrm{~m}$ in dense sand $\left(\phi_{\text {ds-p }}^{\prime}=40^{\circ}, \phi_{\text {ps-p }}^{\prime}=47^{\circ}\right.$, grain size characteristics $\mathrm{D}_{10}=$ $0.3 \mathrm{~mm}, \mathrm{D}_{50}=1 \mathrm{~mm}$ ) having a partially saturated unit weight of $17.5 \mathrm{kN} / \mathrm{m}^{3}$ and a submerged unit weight of $10.7 \mathrm{kN} / \mathrm{m}^{3}$ is subject to vertical downward movement. Calculate the maximum bearing force on the pipe for the ground water table at $0.75 \mathrm{~m}$ and $2.0 \mathrm{~m}$ below the ground surface.

Solution:

1. $\mathrm{H}_{\mathrm{c}} / \mathrm{D}=1.5 \mathrm{~m} / 0.335 \mathrm{~m}=4.5$

2. From Fig. $12 b, \mathrm{~N}_{\mathrm{qVD}}=17.5$ for dense sand, $\mathrm{H}_{\mathrm{c}} / \mathrm{D}=4.5, \mathrm{D}=102 \mathrm{~mm}$. From Fig. 20b, $\mathrm{N}_{\mathrm{qVD}}=13$ for dense sand, $\mathrm{H}_{\mathrm{c}} / \mathrm{D}=4.5, \mathrm{D}=900 \mathrm{~mm}$. Using a simple linear approximation for $\mathrm{H}_{\mathrm{c}} / \mathrm{D}=4.5$ and $\mathrm{D}=0.335 \mathrm{~m}, \mathrm{~N}_{\mathrm{qVD}}=16.2$.

3. For a $0.75-\mathrm{m}$ depth of the ground water table, the effective vertical stress, $\bar{\gamma} H c$ at $\mathrm{H}_{\mathrm{c}}$ of $1.5 \mathrm{~m}=17.5 \mathrm{kN} / \mathrm{m}^{3}(0.75 \mathrm{~m})+10.7 \mathrm{kN} / \mathrm{m}^{3}(0.75 \mathrm{~m})=21.2 \mathrm{kN} / \mathrm{m}^{2}$.

4. From the definition of $\mathrm{N}_{\mathrm{qVD}}=\mathrm{F}_{\mathrm{VD}} /\left(\mathrm{H}_{\mathrm{c}} \bar{\gamma} \mathrm{DL}\right), \mathrm{F}_{\mathrm{VD}}=\mathrm{N}_{\mathrm{qVD}} \mathrm{H}_{\mathrm{c}} \bar{\gamma} \mathrm{DL}=16.2 * 21.2$ $\mathrm{kN} / \mathrm{m}^{2} * 0.335 \mathrm{~m} * 1 \mathrm{~m}=115.1 \mathrm{kN} / \mathrm{m}$, which is the maximum vertical bearing force.

5. For a 2-m depth of ground water table, the effective vertical stress, $\bar{\gamma} H_{c}$ at $\mathrm{H}_{\mathrm{c}}$ of $1.5 \mathrm{~m}=$ $17.5 \mathrm{kN} / \mathrm{m}^{3}(1.5 \mathrm{~m})=26.3 \mathrm{kN} / \mathrm{m}^{2}$. From the definition of $\mathrm{N}_{\mathrm{qVD}}=\mathrm{F}_{\mathrm{VD}} /\left(\mathrm{H}_{\mathrm{c}} \bar{\gamma} \mathrm{DL}\right), \mathrm{F}_{\mathrm{VD}}=$ $\mathrm{N}_{\mathrm{qVD}} \mathrm{H}_{\mathrm{c}} \bar{\gamma} \mathrm{DL}=16.2 * 26.3 \mathrm{kN} / \mathrm{m}^{2} * 0.335 \mathrm{~m} * 1 \mathrm{~m}=142.7 \mathrm{kN} / \mathrm{m}$

6. Because the grain size distribution $\left(D_{10}=0.3 \mathrm{~mm}, \mathrm{D}_{50}=1 \mathrm{~mm}\right)$ falls within the zone in Fig. 21 with low potential for suction, assume a $10 \%$ increase in maximum vertical bearing force: $142.7 \mathrm{kN} / \mathrm{m} * 1.1=157.0 \mathrm{kN} / \mathrm{m}$, which is the maximum vertical bearing force per unit length. 
Example 2: A pipe with an outside diameter of $\mathrm{D}=1220 \mathrm{~mm}$ and depth to pipe centerline of $\mathrm{H}_{\mathrm{c}}$ $=2.5 \mathrm{~m}$. in medium dense sand $\left(\phi_{\text {ds-p }}^{\prime}=35^{\circ}\right.$ and grain size characteristics of $\mathrm{D}_{10}=0.15 \mathrm{~mm}, \mathrm{D}_{50}$ $=0.3 \mathrm{~mm}$ ) having a partially saturated unit weight of $16.6 \mathrm{kN} / \mathrm{m}^{3}$. The pipeline is subject to oblique fault movement with horizontal and vertical components of $2 \mathrm{~m}$ and $1 \mathrm{~m}$, respectively, perpendicular to the pipeline longitudinal axis. Calculate the maximum pipe force on the down thrown side of the fault and a bi-linear relationship for the oblique upward force vs displacement relationship that applies at this location.

\section{Solution:}

1. $\mathrm{H}_{\mathrm{c}} / \mathrm{D}=2.5 \mathrm{~m} / 1.22 \mathrm{~m}=2.0$

2. The angle defining the direction of movement with respect to vertical at the pipe crown: $\beta_{\mathrm{up}}=\tan ^{-1}$ (horizontal component $/$ vertical component $)=\tan ^{-1}(2 \mathrm{~m} / 1 \mathrm{~m})=63.4^{\circ}$

3. From Fig. 9a for medium sand at $\mathrm{H}_{\mathrm{c}} / \mathrm{D}=2.0, \mathrm{~N}_{\mathrm{qVU}}=1.8$. For medium sand the effect of pipe size on soil reaction force for vertical upward movement is negligible.

4. From Fig. $7 \mathrm{a}, \mathrm{N}_{\mathrm{qH}}=7$ for medium sand, $\mathrm{H}_{\mathrm{c}} / \mathrm{D}=2.0, \mathrm{D}=102 \mathrm{~mm}$. The effects of pipe size on $\mathrm{N}_{\mathrm{qH}}$ are negligible for medium sand.

5. From Eq. 11, $\mathrm{i}_{\mathrm{U}}=1+0.25 \beta_{\mathrm{up}} /\left(90-0.75 \beta_{\mathrm{up}}\right) *\left(\mathrm{~N}_{\mathrm{qH}} / \mathrm{N}_{\mathrm{qVU}}-1\right)=1+0.25 * 63.4 /(90-$ $0.75 * 63.4) *(7 / 1.8-1)=2.1$

6. From Eq. 9, $\mathrm{N}_{\mathrm{qOU}}=\mathrm{i}_{\mathrm{U}} \mathrm{N}_{\mathrm{qVU}}=2.1 * 1.8=3.8$

7. The effective vertical stress, $\bar{\gamma} H_{c}$ at $\mathrm{H}_{\mathrm{c}}$ of $2.5 \mathrm{~m}=16.6 \mathrm{kN} / \mathrm{m}^{3}(2.5 \mathrm{~m})=42 \mathrm{kN} / \mathrm{m}^{2}$.

8. From the definition of $\mathrm{N}_{\mathrm{qOU}}=\mathrm{F}_{\mathrm{mOU}} /\left(\mathrm{H}_{\mathrm{c}} \bar{\gamma} \mathrm{DL}\right), \mathrm{F}_{\mathrm{mOU}}=\mathrm{N}_{\mathrm{qOU}} \mathrm{H}_{\mathrm{c}} \bar{\gamma} \mathrm{DL}=3.8 * 42$ $\mathrm{kN} / \mathrm{m}^{2} * 1.22 \mathrm{~m} * 1 \mathrm{~m}=195 \mathrm{kN} / \mathrm{m}$

9. Because the grain size distribution $\left(\mathrm{D}_{10}=0.15 \mathrm{~mm}, \mathrm{D}_{50}=0.3 \mathrm{~mm}\right)$ falls within the zone in Fig. 21 with moderate potential for suction, assume a $50 \%$ increase in maximum 
oblique upward pipe force: $195 \mathrm{kN} / \mathrm{m} * 1.5=293 \mathrm{kN} / \mathrm{m}$. This pipe force can used to assess the sensitivity of pipeline response to suction-enhanced soil reaction.

The bi-linear relationship for the oblique upward force vs displacement is estimated as follows:

1. From Table 3 for medium sand, $\mathrm{Y}_{\mathrm{mOU}} / \mathrm{H}_{\mathrm{c}}=0.017$

2. Applying Eq. 14 with $\mathrm{b}=0.45$ from Table 3 and $\mathrm{N}_{\mathrm{qOU}}=3.8$ from $\# 6$ above: $\mathrm{K}_{70 \mathrm{OU}}=$ $\left(\mathrm{N}_{\mathrm{qOU}} \bar{\gamma} \mathrm{DL}\right) /\left(\mathrm{b}\left[\mathrm{Y}_{\mathrm{mim}} / \mathrm{H}_{\mathrm{c}}\right]\right)=\left(3.8 * 16.6 \mathrm{kN} / \mathrm{m}^{3} * 1.22 \mathrm{~m} * 1 \mathrm{~m}\right) /(0.45[0.017])=10,000 \mathrm{kN} / \mathrm{m} / \mathrm{m}$ for dry sand.

3. To estimate the stiffness for partially saturated conditions: $2\left(\mathrm{~K}_{700 \mathrm{O}}\right.$ for dry sand $)=$ $2(10,000 \mathrm{kN} / \mathrm{m})=20,000 \mathrm{kN} / \mathrm{m} / \mathrm{m}$.

4. The bi-linear force vs displacement relationship for oblique uplift increases linearly with a slope of $20,000 \mathrm{kN} / \mathrm{m} / \mathrm{m}$ to a maximum force $=293 \mathrm{kN} / \mathrm{m}$, which is the estimated maximum oblique force that accounts for suction from \# 9 above.

\section{Conclusions}

The methodology presented in this paper is based on large-scale test results to 1) provide core information on parameters for analysis and design, and 2) validate FE models for systematic numerical simulation of soil-pipe interaction under conditions that extend beyond those addressed by the large-scale experiments. The resulting methodology and dimensionless plots are applicable for underground pipelines and conduits at any depth, subject to relative soil movement in any direction in medium to very dense sands for dry/saturated conditions. Guidance is provided for estimating the general effects of suction for pipelines in partially saturated sands. Specific findings of interest are:

- The maximum downward pipe force from FE analyses in dry/saturated sand is about onethird the maximum force determined with conventional bearing capacity equations (e.g., 
Vesić 1975; Kulhawy et al. 1983). The maximum vertical pipe forces currently recommended in design guidelines appear to be overly conservative.

- The dimensionless maximum soil-pipe reaction force in any direction normalized by the dimensionless maximum horizontal reaction force, $\mathrm{N}_{\mathrm{q}} / \mathrm{N}_{\mathrm{qH}}$, has a unique value for a given $\mathrm{H}_{\mathrm{c}} / \mathrm{D}$ and direction of pipe movement, which does not depend on sand density and associated friction angles. Experimental and analytical results normalized with respect to maximum lateral force are therefore provided on $360^{\circ}$ plots to predict maximum pipe loads for any movement direction at any dimensionless depth, $\mathrm{H}_{\mathrm{c}} / \mathrm{D}$.

- Pipe size affects the dimensionless soil reaction force at constant $H_{c} / D$ for vertical downward and lateral displacement of the pipe in dry dense and very dense sand because increasing $\mathrm{D}$ under these conditions increases the confining stress at depth, thereby decreasing dilatant behavior. No significant effect of pipe size is observed on soil reaction force for vertical upward movement of the pipe.

- A method for checking the potential for suction-enhanced soil reaction forces in sand is proposed on the basis of soil grain size characteristics. In some instances, lateral soil-pipe forces in partially sand may be larger than twice those in dry sand.

The analytical results for pipeline response to strike-slip fault rupture using the proposed methodology compare favorably with the results of large-scale tests in which strike-slip fault movement was imposed on 250-mm and 400-mm-diameter HDPE pipelines in partially saturated sand. In general, the methodology provides results appropriate for soil-pipeline interaction analysis and design, provided that localized, secondary soil movements are accounted for and that $3 \mathrm{D}$ deformation, including ovaling and shell buckling of the pipe, are addressed with supplemental analysis and design measures. 
The analytical horizontal and vertical uplift soil-pipe forces have been validated by large-scale test results. Large-scale tests are highly recommended to qualify vertical soil-pipe bearing forces, which are shown analytically in this work to be significantly lower than those given by conventional bearing capacity equations. Tests are also recommended to quantify further the effect of increasing diameter on the reduction of dimensionless soil-pipe forces, especially for vertical downward movement, and to provide guidance for treating pipelines greater than $900 \mathrm{~mm}$ in diameter.

\section{Acknowledgements}

The work on which this paper was based was supported by the George E. Brown, Jr. Network for Earthquake Engineering Simulation (NEES) Program of the National Science Foundation (NSF) under Grant No. CMMI-1041498. Any opinions, findings and conclusions or recommendations expressed in this material are those of the authors and do not necessarily reflect the views of the NSF. 


\section{REFERENCES}

ABAQUS. 2014. “Theory Manual of ABAQUS,” ABAQUS, Inc., Providence, RI.

Akbas, S. O., and Kulhawy, F. H. 2009a. Axial compression of footings in cohesionless soils. I: Load-settlement behavior. Journal of geotechnical and geoenvironmental engineering, 135(11), 1562-1574.

Akbas, S. O., and Kulhawy, F. H. 2009b. Axial compression of footings in cohesionless soils. II: Bearing Capacity. Journal of geotechnical and geoenvironmental engineering, 135(11), 1575 1582.

ALA. 2005. Seismic Guidelines for Water Pipelines. American Lifelines Alliance http://www.americanlifelinesalliance.com/pdf/SeismicGuidelines_WaterPipelines_Comm.pdf

Anastasopoulos, I., Gazetas, G., Bransby, M. F., Davies, M. C. R., and El Nahas, A. 2007. Fault rupture propagation through sand: Finite-element analysis and validation through centrifuge experiments. Journal of Geotechnical and Geoenvironmental Engineering, 133(8), 943-958.

ASCE. 1984. Guidelines for the seismic design of oil and gas pipeline systems. Committee on Gas and Liquid Fuel Lifelines, American Society of Civil Engineers, Reston, VA

ASTM D 2321-11 2011. Standard practice for underground installation of thermoplastic pipe for sewers and other gravity-flow application, American Society for Testing and Materials.

Bolton, M.D. 1986. "The Strength and Dilatancy of Sands.” Geotechnique, 36(1): 65-78.

Bouziou, D. 2015. Earthquake-induced ground deformation effects on buried pipelines, Ph.D Thesis, Cornell University, Ithaca, NY, January.

Davis, E. H. 1968. Theories of plasticity and the failure of soil masses. Soil mechanics: Selected topics, 341-380.

Dickin, E.A. 1994. Uplift resistance of buried pipelines in sand. Soils and Foundations, 34(2): 41-48. doi:10.3208/sandf1972.34.2_41.

Duncan, J. M., and Chang, C. Y. 1970. Nonlinear analysis of stress and strain in soils. Journal of the Soil Mechanics and Foundations Division, 96(5), 1629-1653.

Hirany, A., and Kulhawy, F. H. 2002. On the interpretation of drilled foundation load test results. In Deep Foundations 2002@sAn International Perspective on Theory, Design, Construction, and Performance (pp. 1018-1028). ASCE.

Honegger, D., and Nyman, D.J. 2004. "Guidelines for the Seismic Design and Assessment of Natural Gas and Liquid Hydrocarbon Pipelines," Pipeline Research Council International, Catalog No. L51927, October. 
Huang, W., Nübel, K., and Bauer, E. 2002. Polar extension of a hypoplastic model for granular materials with shear localization. Mechanics of Materials, 34(9), 563-576.

Janbu, N. 1963. Soil compressibility as determined by oedometer and triaxial tests. Proc. ECSMFE Wiesbaden, 1, 19-25.

Jefferies, M. G. 1993. Nor-Sand: a simple critical state model for sand. Geotechnique, 43(1), 91103.

Jung, J. K. 2010. Soil-pipe interaction under plane strain conditions. Ph.D Thesis, Cornell University, Ithaca, NY, August

Jung, J. K., O’Rourke, T. D., and Olson, N. A. 2013a. Uplift soil-pipe interaction in granular soil. Canadian Geotechnical Journal, 50(7), 744-753.

Jung, J. K., O’Rourke, T. D., and Olson, N. A. 2013b. Lateral soil-pipe interaction in dry and partially saturated sand. Journal of Geotechnical and Geoenvironmental Engineering, 139(12), 2028-2036.

Kulhawy, F. H., O'Rourke, T. D., Stewart, J. P., and Beech, J. F. 1983. Transmission Line Structure Foundations for Uplift-compression Loading, Load Test Summaries: Appendix to EPRI Final Report EL-2870. Electric Power Research Institute.

Lings, M. L., and Dietz, M. S. 2004. An improved direct shear apparatus for sand. Geotechnique, 54(4), 245-256.

Merry, S. M., and Bray, J. D. 1997. Time-dependent mechanical response of HDPE geomembranes. Journal of geotechnical and geoenvironmental engineering, 123(1), 57-65.

Meyerhof, G. G. 1973. The uplift capacity of foundations under oblique loads. Canadian Geotechnical Journal, 10(1), 64-70.

Mühlhaus, H. B., and Vardoulakis, I. 1987. The thickness of shear bands in granular materials. Geotechnique, 37(3), 271-283.

Nyman, K. J. 1984. Soil response against oblique motion of pipes. Journal of Transportation Engineering, 110(2), 190-202.

Olson, N. 2009. Soil performance for large scale soil-pipeline tests. Ph.D Thesis, Cornell University, Ithaca, NY, May

O'Rourke, T. D. 2010. Geohazards and large, geographically distributed systems. Geotechnique, $60(7), 505-543$. 
O'Rourke, T.D., Druschel, S.J., and Netravali A.N. 1990. Shear strength characteristics of sandpolymer interfaces," Journal of Geotechnical Engineering, ASCE, 116(3), 451-469.

O’Rourke, T.D., Jung, J.K., and Argyrou, C. 2015. Underground infrastructure response to earthquake-induced ground deformation. Proceedings $6^{\text {th }}$ Intl. Conf. Earthquake Geotech. Engr., Intl. Soc. Soil Mech. and Found. Engr., Christchurch, NZ.

O’Rourke, T. D., Jezerski, J. M., Olson, N. A., Bonneau, A. L., Palmer, M. C., Stewart, H. E., and Abdoun, T. 2008. Geotechnics of pipeline system response to earthquakes. Geotechnical Earthquake Engineering and Soil Dynamics IV (GEESD), Sacramento, CA, May.

Ovesen, N.K. 1964. Anchor Slabs, Calculation Methods and Model Tests. Bulletin 16, Danish Geotechnical Institute, Copenhagen, Denmark.

Perkins, S. W., and Madson, C. R. 1996. Mechanical and load-settlement characteristics of two lunar soil simulants. Journal of Aerospace Engineering, 9(1), 1-9.

Robert, D.J., and Soga, K. 2009. "Simulations of Soil-Pipeline Interactions in Unsaturated Soils.” Report to Tokyo Gas Co. Ltd., Cambridge University.

Robert, D.J., Soga, K., O’Rourke, T.D., and Sakanoue, T. 2016a. "Pipelines subjected to fault movement in dry and unsaturated soils." International Journal of Geomechanics, ASCE, accepted for publication.

Robert, D.J., Soga, K., and O’Rourke, T.D. 2016b. Lateral load-displacement behavior of pipelines in unsaturated sands. Journal of Geotechnical and Geoenvironmental Engineering, ASCE, accepted for publication.

Roscoe, K. H. 1970. The influence of strains in soil mechanics. Geotechnique, 20(2), 129-170.

Rowe, R. K., and Davis, E. H. 1982. Behaviour of anchor plates in sand. Geotechnique, 32(1), $25-41$.

Shiraishi, S. 1990. Variation in bearing capacity factors of dense sand assessed by model loading tests. Soils and Foundations, 30(1), 17-26.

Trautmann, C. H., and O'Rourke, T. D. 1985. Lateral force-displacement response of buried pipe. Journal of Geotechnical Engineering, 111(9), 1077-1092.

Trautmann, C. H., O'Rourfce, T. D., and Kulhawy, F. H. 1985. Uplift force-displacement response of buried pipe. Journal of Geotechnical Engineering, 111(9), 1061-1076.

Turner, J.E. 2004. Lateral Force - Displacement Behavior of Pipes in Partially Saturated Sand, M.S. Thesis, Cornell University, Ithaca, NY, May 
Vardoulakis, I., and Graf, B. 1985. Calibration of constitutive models for granular materials using data from biaxial experiments. Géotechnique, 35(3), 299-317.

Vazouras, P., Dakoulas, P., and Karamanos, S. A. 2015. Pipe-soil interaction and pipeline performance under strike-slip fault movements. Soil Dynamics and Earthquake Engineering, 72, 48-65.

Vesić, A.S 1975. Bearing capacity of shallow foundations. Chapter 3 in Foundation Engineering Handbook, Ed. by H. Winterkorn and H.Y. Feng, Van Nostrand Reinhold Company, New York, NY, 1975, pp. 212-147.

White, D. J., Teh, K. L., Leung, C. F., and Chow, Y. K. 2008. A comparison of the bearing capacity of flat and conical circular foundations on sand. Géotechnique, 58(10), 781-792.

Wong, K. S., and Duncan, J. M. 1974. Hyperbolic stress-strain parameters for nonlinear finite element analyses of stresses and movements in soil masses. College of Engineering, Office of Research Services, University of California.

Xie, X., Symans, M. D., O'Rourke, M. J., Abdoun, T. H., O'Rourke, T. D., Palmer, M. C., and Stewart, H. E. 2013. Numerical modeling of buried HDPE pipelines subjected to normal faulting: a case study. Earthquake Spectra, 29(2), 609-632.

Yimsiri, S., Soga, K., Yoshizaki, K., Dasari, G. R., and O'Rourke, T. D. 2004. Lateral and upward soil-pipeline interactions in sand for deep embedment conditions. Journal of geotechnical and geoenvironmental engineering, 130(8), 830-842.

Yoshimi, Y., and Kishida, T. 1983, February. Ring torsion apparatus for evaluating friction between soil and metal surfaces: Geotech Test J, V4, N4, Dec 1981, P145-152. In International Journal of Rock Mechanics and Mining Sciences \& Geomechanics Abstracts (Vol. 20, No. 1, p. A7). Pergamon.

Zienkiewicz, O. C., Emson, C., and Bettess, P. 1983. A novel boundary infinite element. International Journal for Numerical Methods in Engineering, 19(3), 393-404.

Zienkiewicz, O. C., Humpheson, C., and Lewis, R. W. 1975. Associated and non-associated visco-plasticity and plasticity in soil mechanics. Geotechnique, 25(4), 671-689. 
Figure Captions

Figure 1. a) Schematic of FE model and (b) bilinear force vs. displacement relationships at pipesoil interface [after Bouziou 2015]

Figure 2. Mohr Coulomb Stress Parameters for a c - $\phi$ Material (Not to Scale) (after Jung, et al. 2013b)

Figure 3. Grain Size Distributions Sands Used in Large-Scale Soil-Pipe Interaction Tests.

Figure 4. $\psi_{\mathrm{p}}$ and $\phi^{\prime}{ }_{\text {ds-p }}$ vs. $\gamma_{\mathrm{d}}$ for Dry RMS Graded Sand at $\sigma^{\prime}{ }_{\mathrm{N}}=2.1 \mathrm{kPa}$ (after Olson (2009))

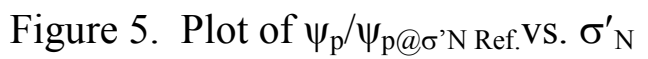

Figure 6. Comparison of Large-scale Test and Analytical Results for Lateral and Upward

Movement of Pipe in Dry and Partially Saturated Sand (after Jung et al. 2013a and 2013b)

Figure 7. Plot of $\mathrm{N}_{\mathrm{qH}}$ vs. $\mathrm{H}_{\mathrm{c}} / \mathrm{D}$ for Dry Medium, Dense, Very Dense Sand, and Partially Saturated Sand for $(a) H_{c} / D \leq 11$, and (b) $H_{c} / D \leq 100$ (After Jung et al. 2013b)

Figure 8. Comparison of Dimensionless Horizontal Force vs Displacement Relationships with Those Provided by ASCE (1984) and ALA (2005)

Figure 9. Plot of $\mathrm{N}_{\mathrm{qVU}}$ vs. $\mathrm{H}_{\mathrm{c}} / \mathrm{D}$ for Dry Medium, Dense, and Very Dense Sand for $(\mathrm{a}) \mathrm{H}_{\mathrm{c}} / \mathrm{D} \leq 13$, and (b) $\mathrm{H}_{\mathrm{c}} / \mathrm{D} \leq 100$

Figure 10. Geometry of the Finite Element Simulations for Vertical Downward and Oblique Pipe Movement

Figure 11. Typical Load-Settlement Curve of Axial Compression Test on Foundation

Figure 12. Summary Plot of $\mathrm{N}_{\mathrm{qVD}}$ vs. $\mathrm{H}_{\mathrm{c}} / \mathrm{D}$ for Vertical Downward Pipe Movement for (a) $\mathrm{H}_{\mathrm{c}} / \mathrm{D}$ $\leq 100$, and $(\mathrm{b}) \mathrm{H}_{\mathrm{c}} / \mathrm{D} \leq 11$

Figure 13. Comparison of $\mathrm{Q}_{\mathrm{tc}-\mathrm{fea}}$ and $\mathrm{Q}_{\mathrm{tc}-\mathrm{bc}}$

Figure 14. Dimensionless Maximum Oblique Downward Force as a Function of Dimensionless Depth for (a) $\mathrm{H}_{\mathrm{c}} / \mathrm{D} \leq 100$, and (b) $\mathrm{H}_{\mathrm{c}} / \mathrm{D} \leq 11$

Figure 15. Dimensionless Maximum Oblique Upward Force as a Function of Dimensionless Depth for $(a) H_{c} / D \leq 100$, and (b) $H_{c} / D \leq 11$

Figure 16. Angle Defining the Direction of Movement with Respect to Vertical at the Pipe Crown $\left(\beta_{\text {up }}\right)$ and at the Pipe Invert $\left(\beta_{\text {down }}\right)$

Figure 17. Comparison of (a) $\mathrm{N}_{\mathrm{qOD}-\mathrm{FE}}$ vs. $\mathrm{N}_{\mathrm{qOD}-\mathrm{s}}$ for $\beta_{\text {down }}=45^{\circ}$, (b) $\mathrm{N}_{\mathrm{qOU}-F E}$ Vs. $\mathrm{N}_{\mathrm{qOU}-\mathrm{s}}$ for $\beta_{\text {up }}=$ $45^{\circ}$, (c) $\mathrm{N}_{\mathrm{qOD}-\mathrm{FE}} \mathrm{Vs} . \mathrm{N}_{\mathrm{qOD}-\mathrm{s}}$ for $\beta_{\text {down }}=22.5^{\circ}$, and (d) $\mathrm{N}_{\mathrm{qOU}-\mathrm{FE}} \mathrm{Vs} . \mathrm{N}_{\mathrm{qOU}-\mathrm{s}}$ for $\beta_{\text {up }}=22.5^{\circ}$ 
Figure 18. Visualization of $\mathrm{N}_{\mathrm{q}} / \mathrm{N}_{\mathrm{qH}}$ for $\mathrm{H}_{\mathrm{c}} / \mathrm{D}=3.5-11$

Figure 19. Visualization of $\mathrm{N}_{\mathrm{q}} / \mathrm{N}_{\mathrm{qH}}$ for $\mathrm{H}_{\mathrm{c}} / \mathrm{D}=15-100$

Figure 20. Plot of (a) $\mathrm{N}_{\mathrm{qH}}$ vs. $\mathrm{H}_{\mathrm{c}} / \mathrm{D}$ and (b) $\mathrm{N}_{\mathrm{qVD}}$ vs. $\mathrm{H}_{\mathrm{c}} / \mathrm{D}$ for Various Pipe Diameters

Figure 21. Suction Effects in Sand Related to Grain Size Distribution (after Robert et al. 2016)

Figure 22. Hyperbolic and Bi-Linear Force vs. Displacement Relationship of (a) Generic Form, and (b) Horizontal Pipe Movement

Figure 23. Large-Scale Split-Basin Test Facility at the Cornell University.

Figure 24. Measured and Numerically Simulated Strains for Large-Scale Test of Strike-Slip Faulting on a) 250-mm and b) 400-mm HDPE Pipelines

Figure 25. Flowchart for Estimation of Maximum Soil-Pipe Reaction Force 


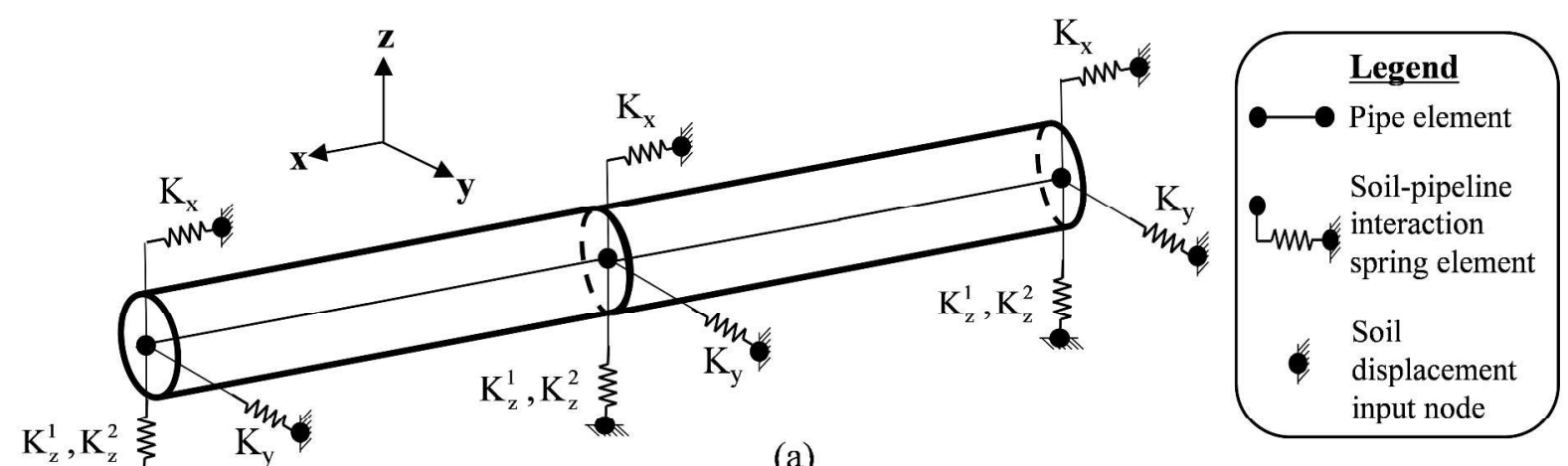

(a)
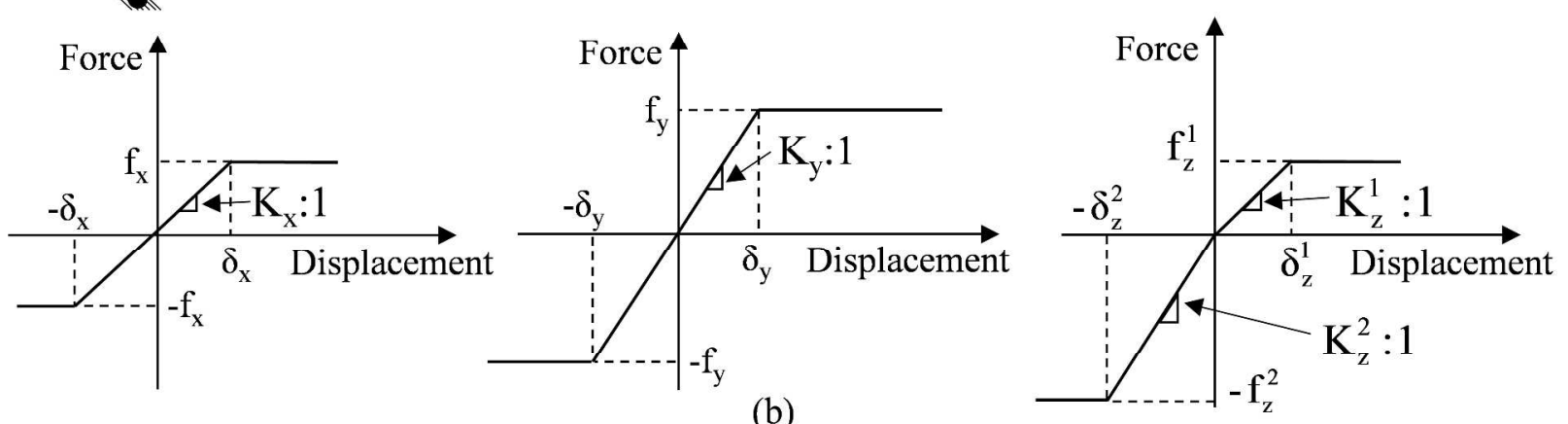

Figure 1. a) Schematic of FE model and (b) bilinear force vs. displacement relationships at pipesoil interface [after Bouziou 2015]

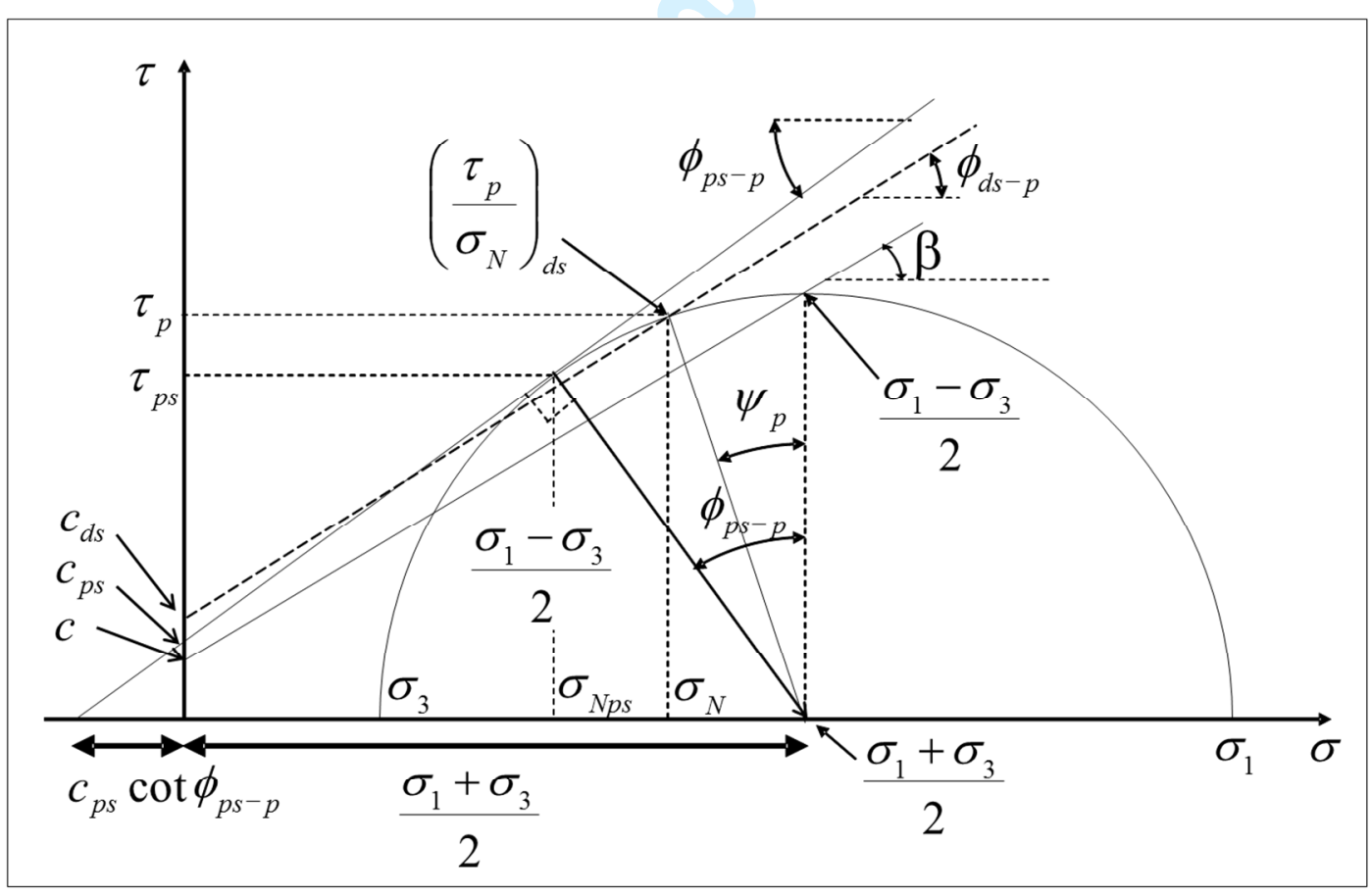

Figure 2. Mohr Coulomb Stress Parameters for a c - $\phi$ Material (Not to Scale) (after Jung, et al. 2013b) 


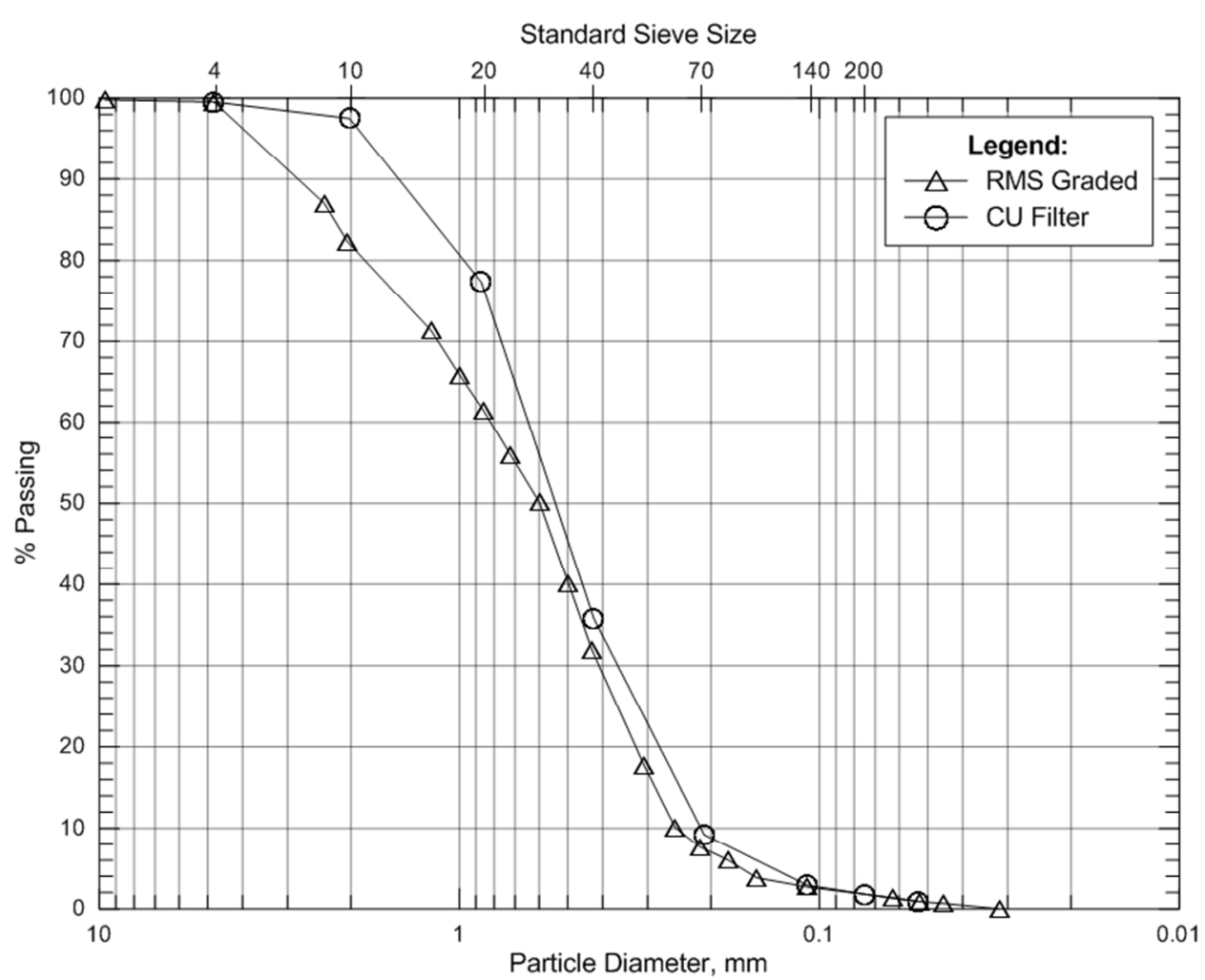

Figure 3. Grain Size Distributions Sands Used in Large-Scale Soil-Pipe Interaction Tests.

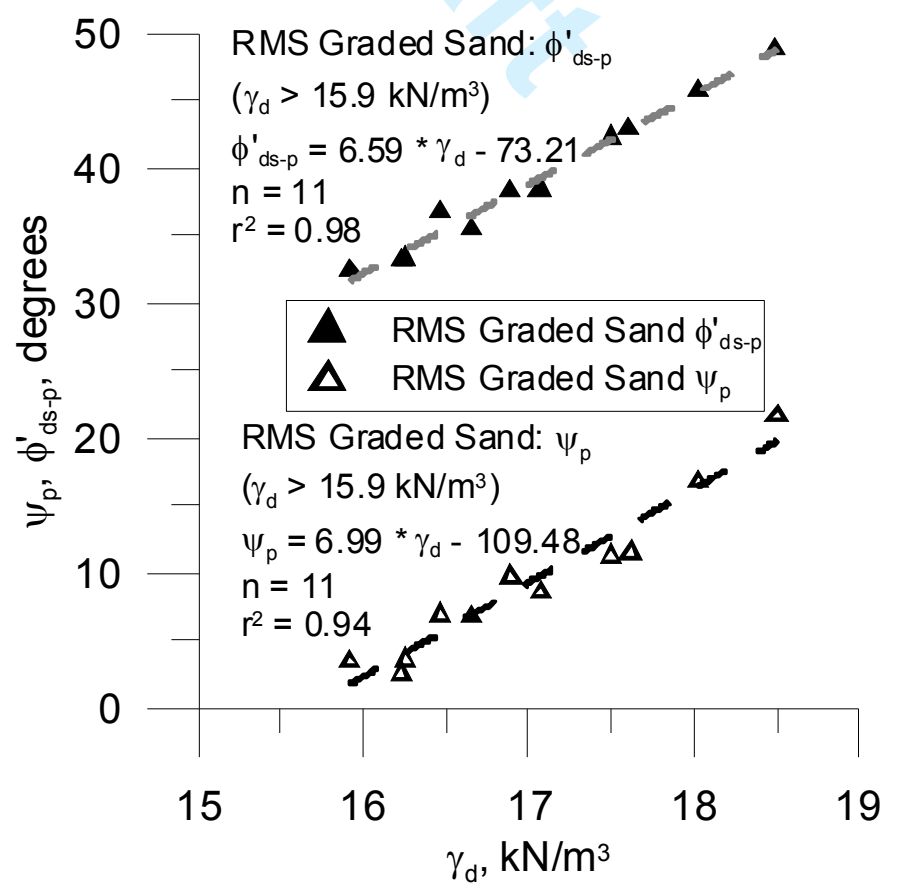

Figure 4. $\psi_{\mathrm{p}}$ and $\phi^{\prime}{ }_{\text {ds-p }}$ vs. $\gamma_{\mathrm{d}}$ for Dry RMS Graded Sand at $\sigma^{\prime}{ }_{\mathrm{N}}=2.1 \mathrm{kPa}$ (after Olson (2009)) 


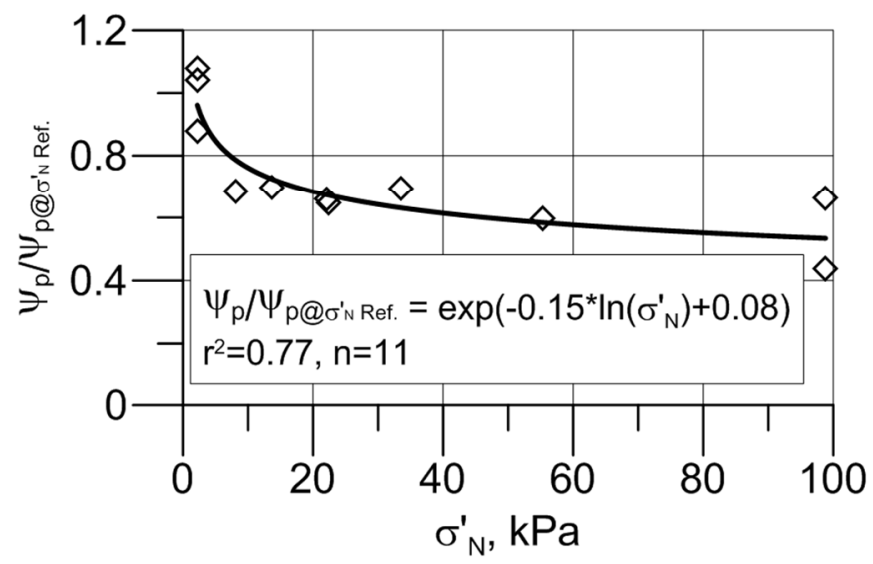

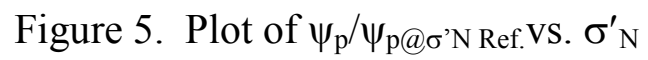
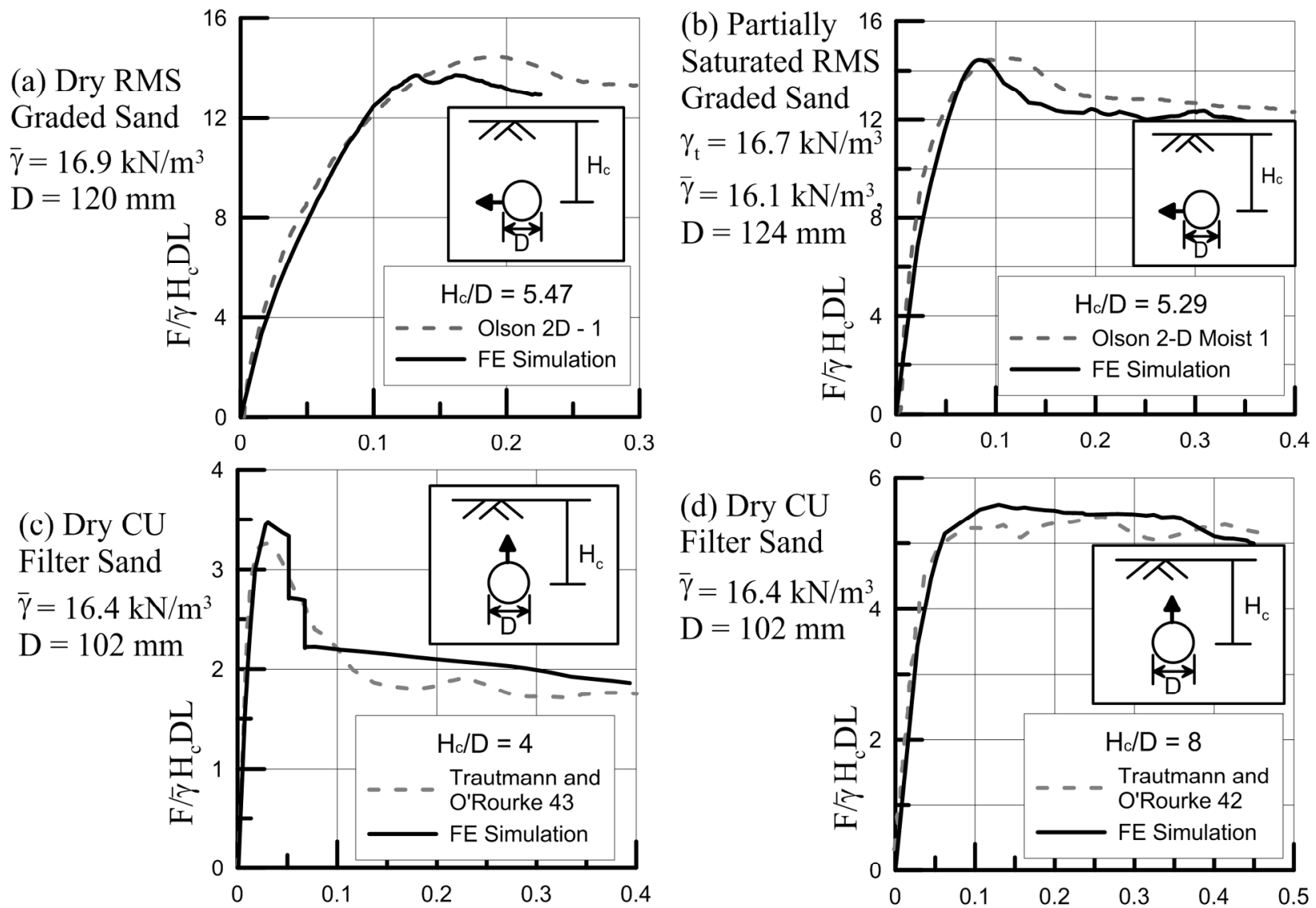

Dimensionless Displacement

Dimensionless Displacement

Figure 6. Comparison of Large-scale Test and Analytical Results for Lateral and Upward Movement of Pipe in Dry and Partially Saturated Sand (after Jung et al. 2013a and 2013b) 

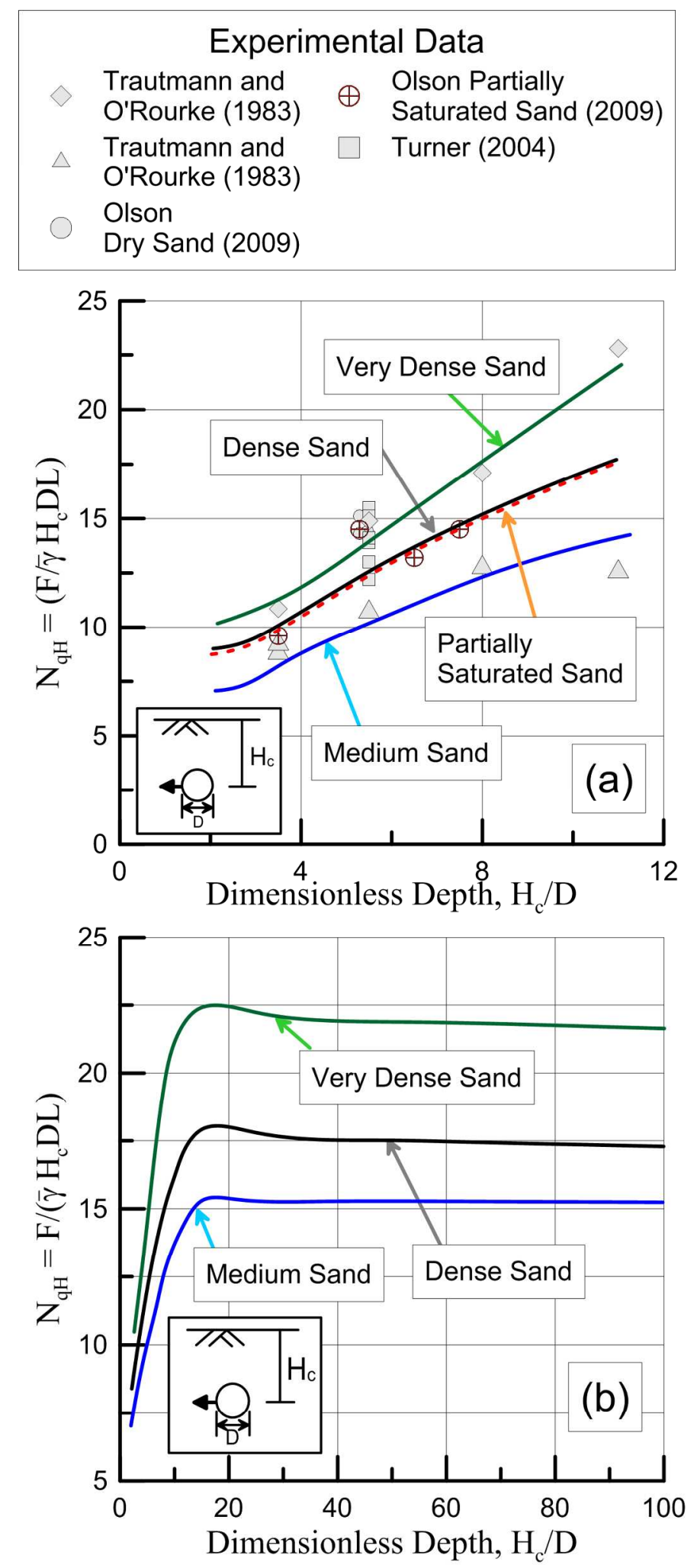

Figure 7. Plot of $\mathrm{N}_{\mathrm{qH}}$ vs. $\mathrm{H}_{\mathrm{c}} / \mathrm{D}$ for Dry Medium, Dense, Very Dense Sand, and Partially Saturated Sand for (a) $H_{c} / D \leq 11$, and (b) $H_{c} / D \leq 100$ (After Jung et al. 2013b) 


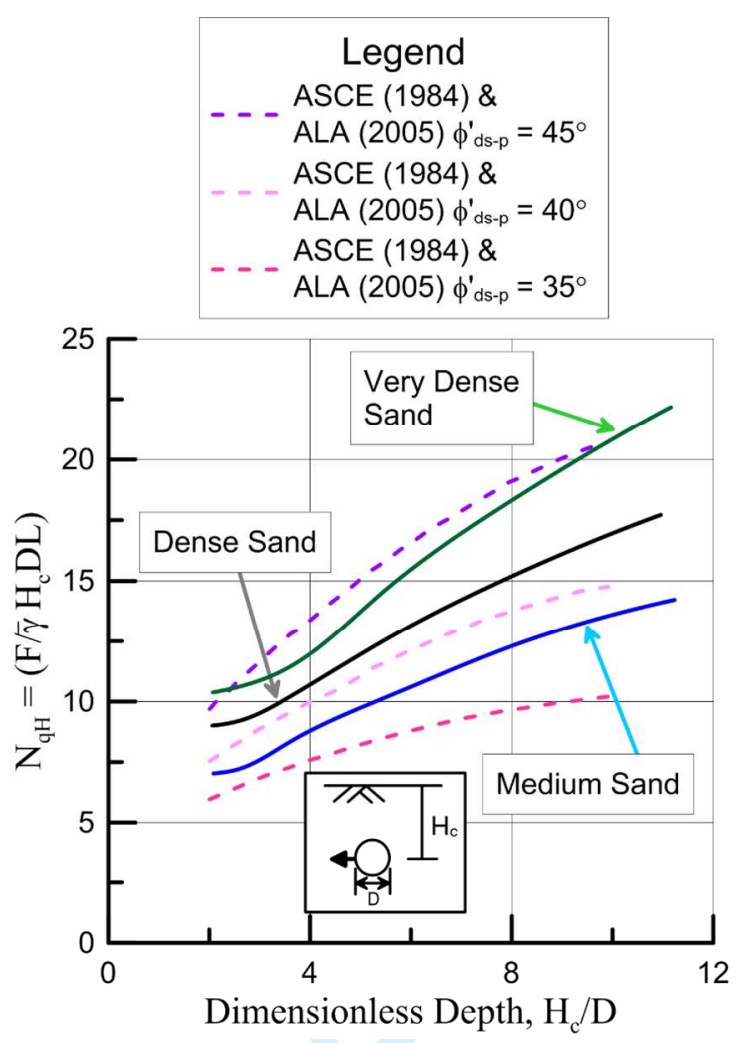

Figure 8. Comparison of Dimensionless Horizontal Force vs Displacement Relationships with Those Provided by ASCE (1984) and ALA (2005) 

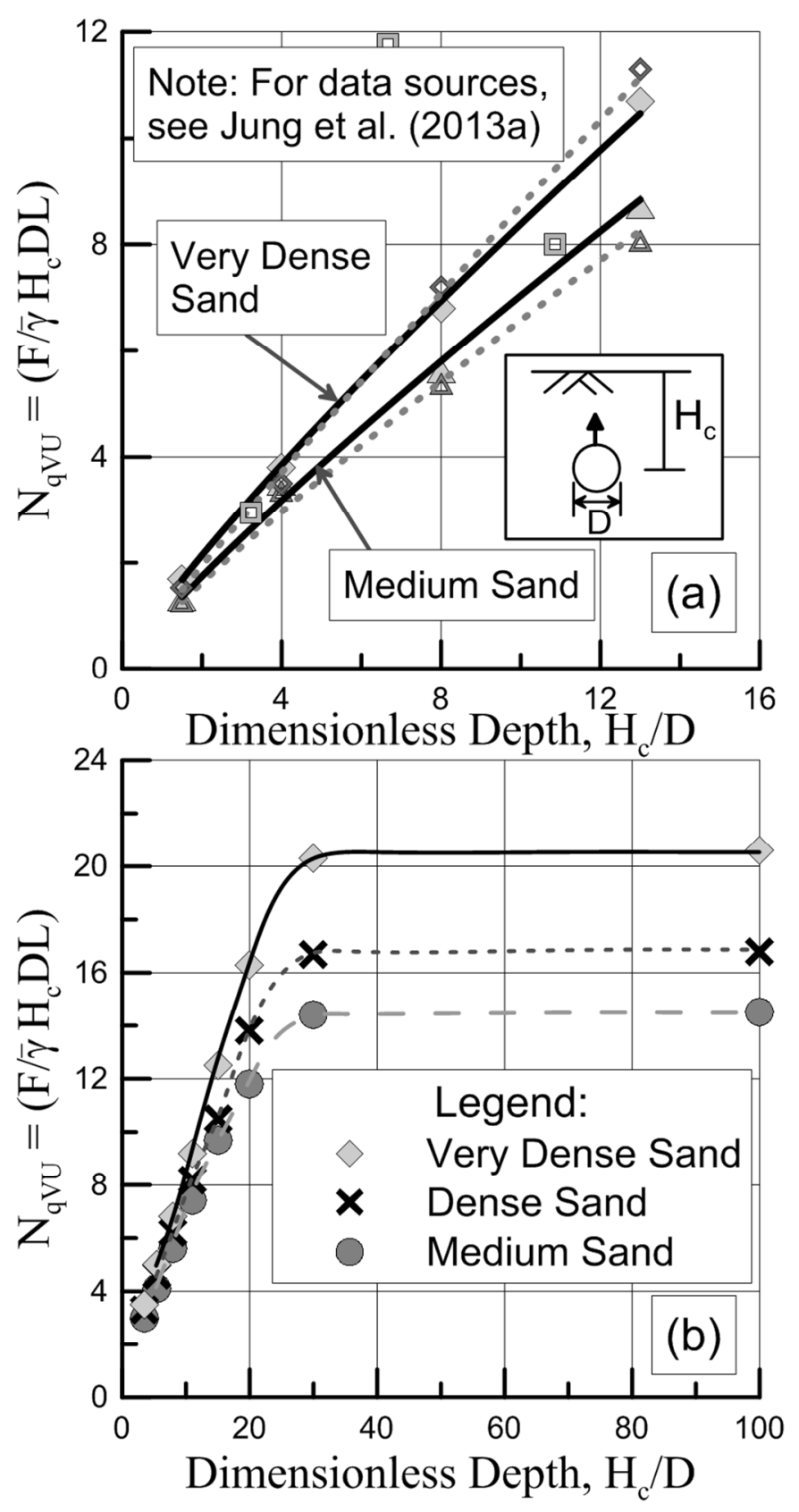

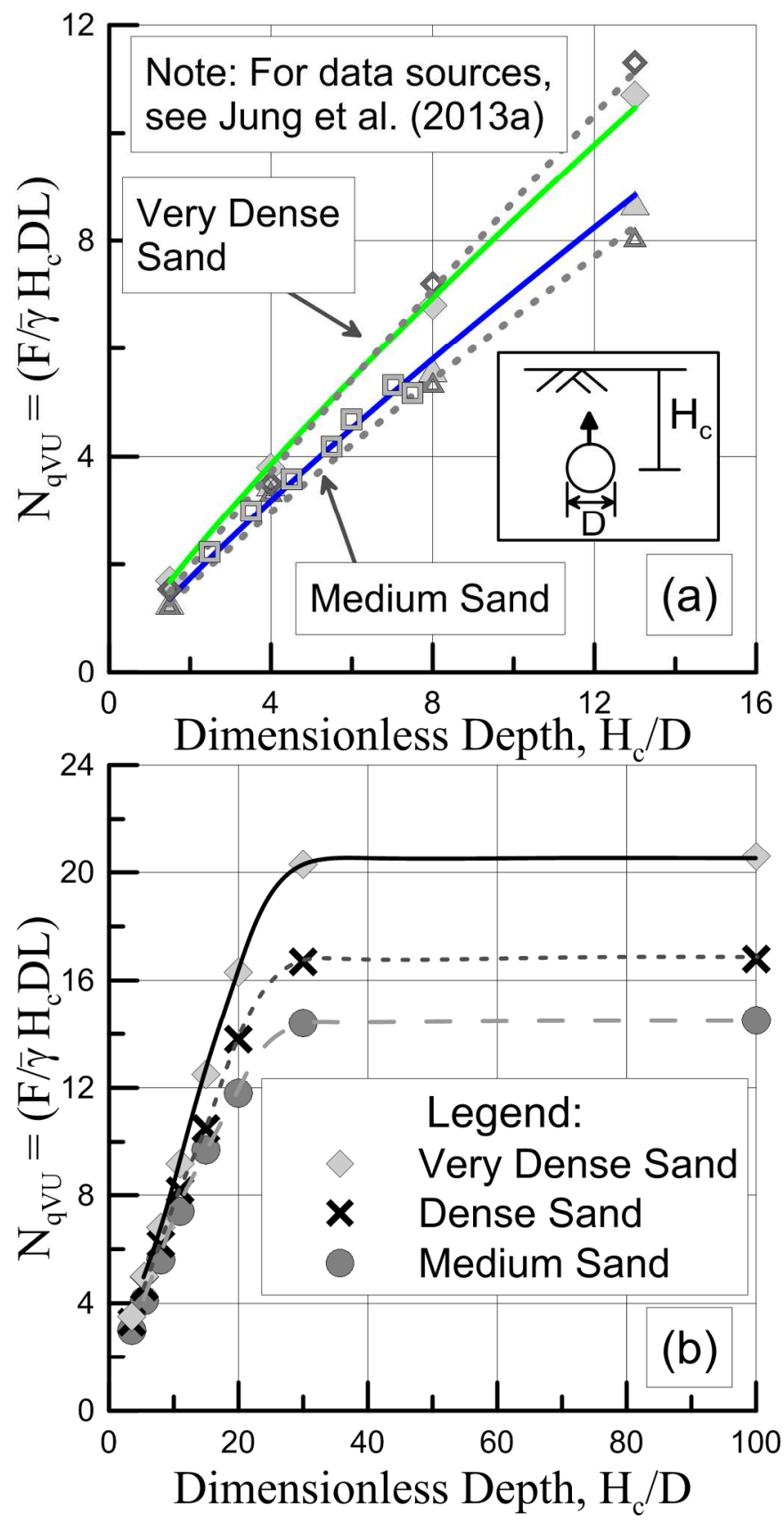

Figure 9. Plot of $\mathrm{N}_{\mathrm{qVu}}$ vs. $\mathrm{H}_{\mathrm{c}} / \mathrm{D}$ for Dry Medium, Dense, and Very Dense Sand for $(\mathrm{a}) \mathrm{H}_{\mathrm{c}} / \mathrm{D} \leq 13$, and (b) $\mathrm{H}_{\mathrm{c}} / \mathrm{D} \leq 100$ 


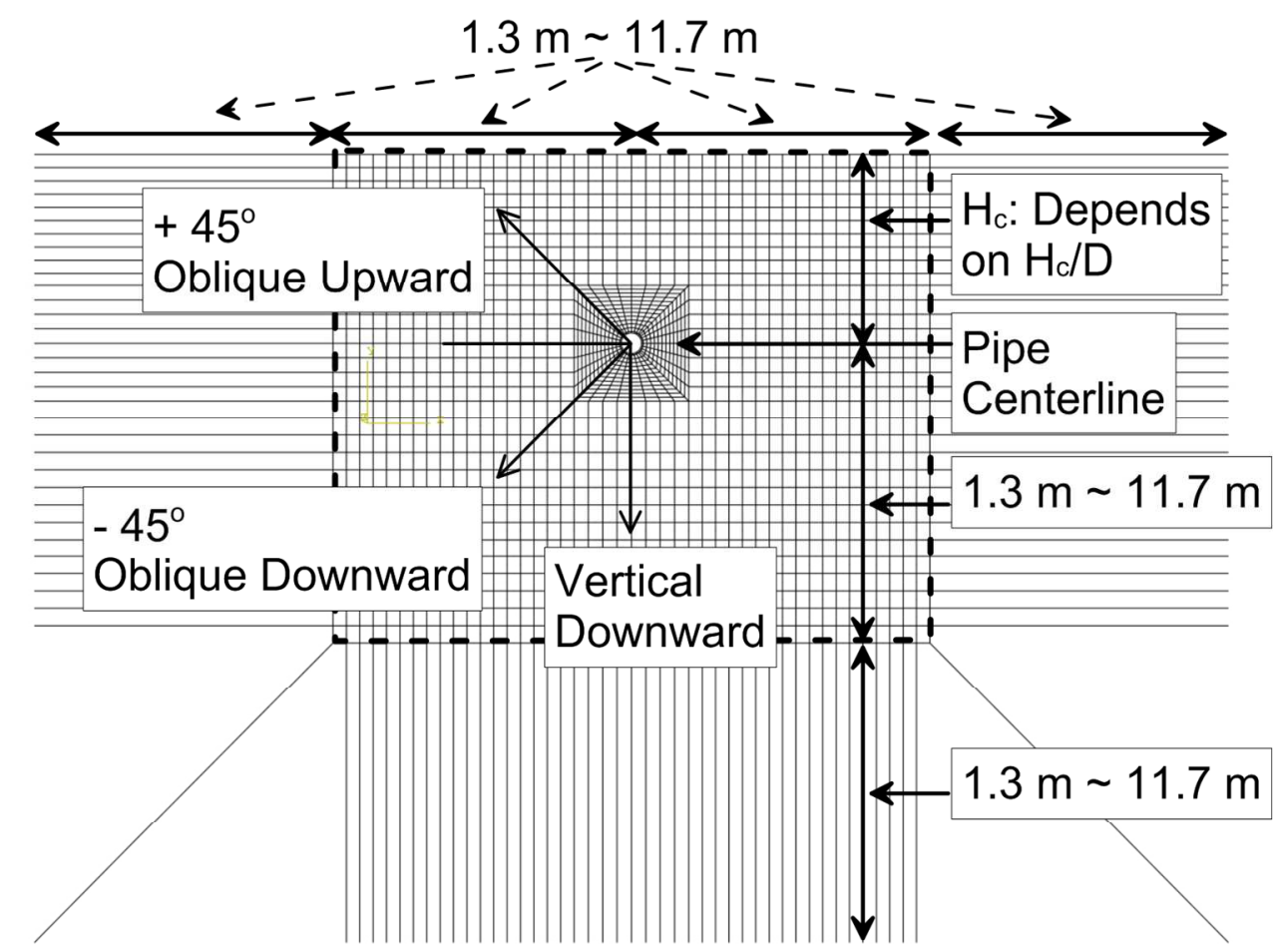

Figure 10. Geometry of the Finite Element Simulations for Vertical Downward and Oblique Pipe Movement

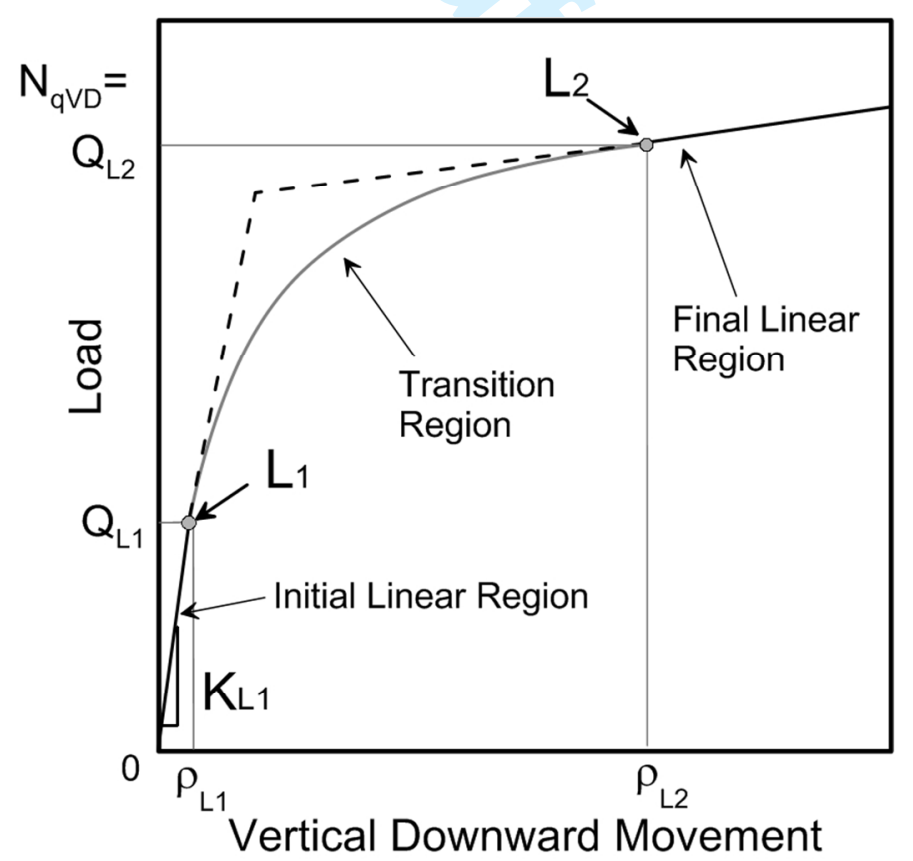

Figure 11. Typical Load-Settlement Curve of Axial Compression Test on Foundation 

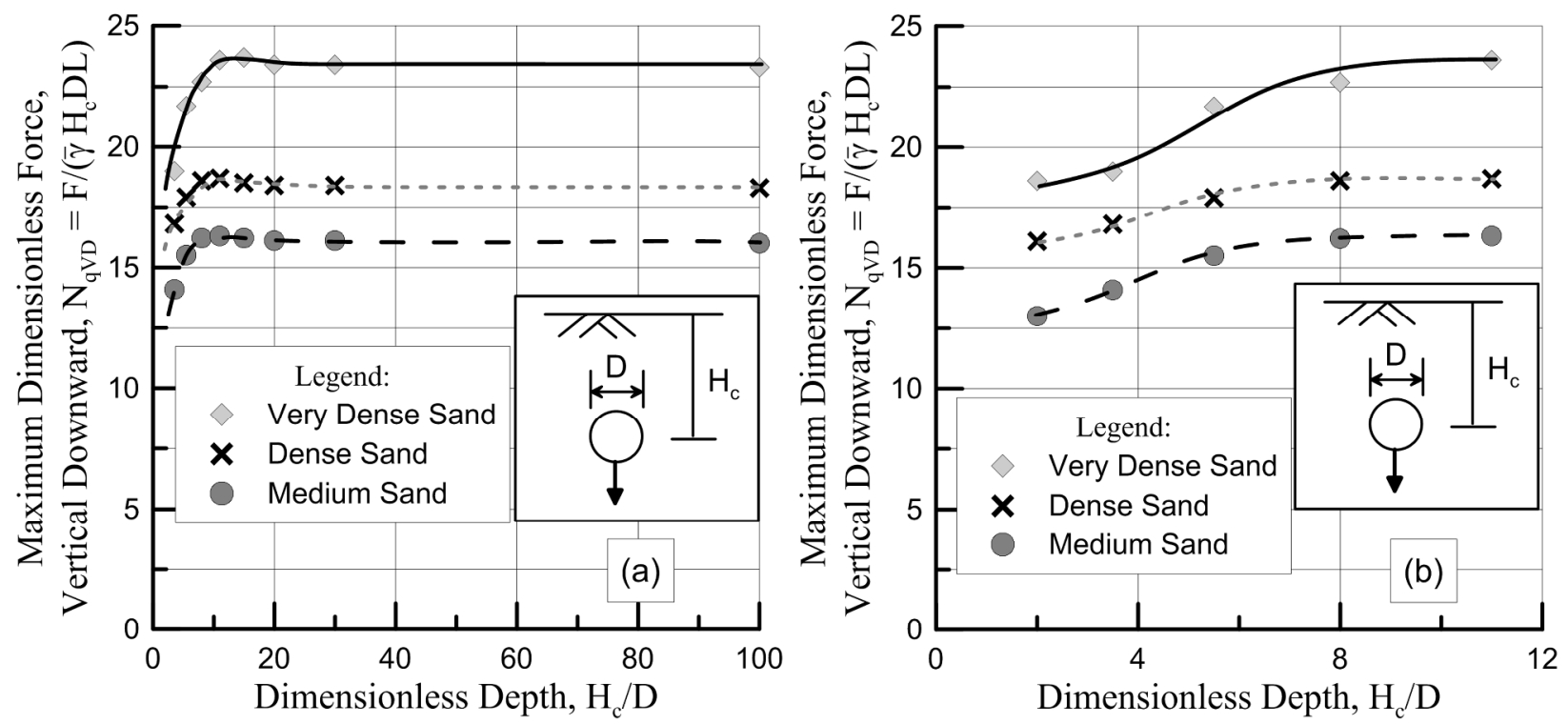

Figure 12. Summary Plot of $\mathrm{N}_{\mathrm{qVD}}$ vs. $\mathrm{H}_{\mathrm{c}} / \mathrm{D}$ for Vertical Downward Pipe Movement for (a) $\mathrm{H}_{\mathrm{c}} / \mathrm{D}$ $\leq 100$, and $(\mathrm{b}) \mathrm{H}_{\mathrm{c}} / \mathrm{D} \leq 11$

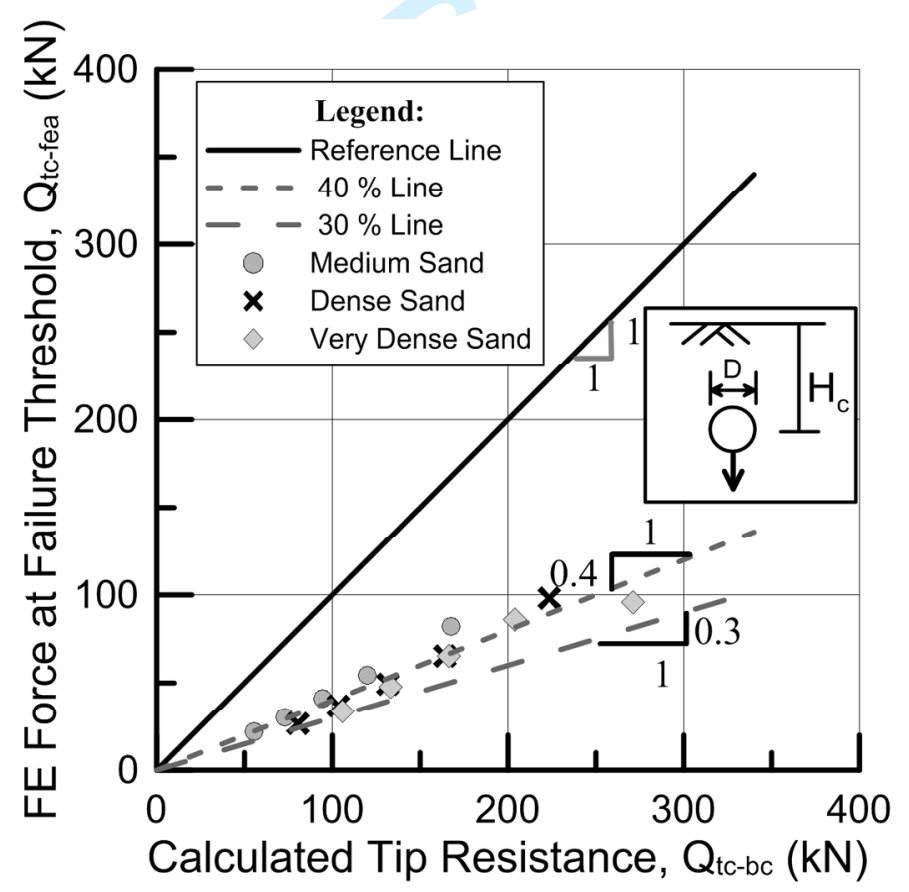

Figure 13. Comparison of $\mathrm{Q}_{\mathrm{tc}-\mathrm{fea}}$ and $\mathrm{Q}_{\mathrm{tc}-\mathrm{bc}}$ 

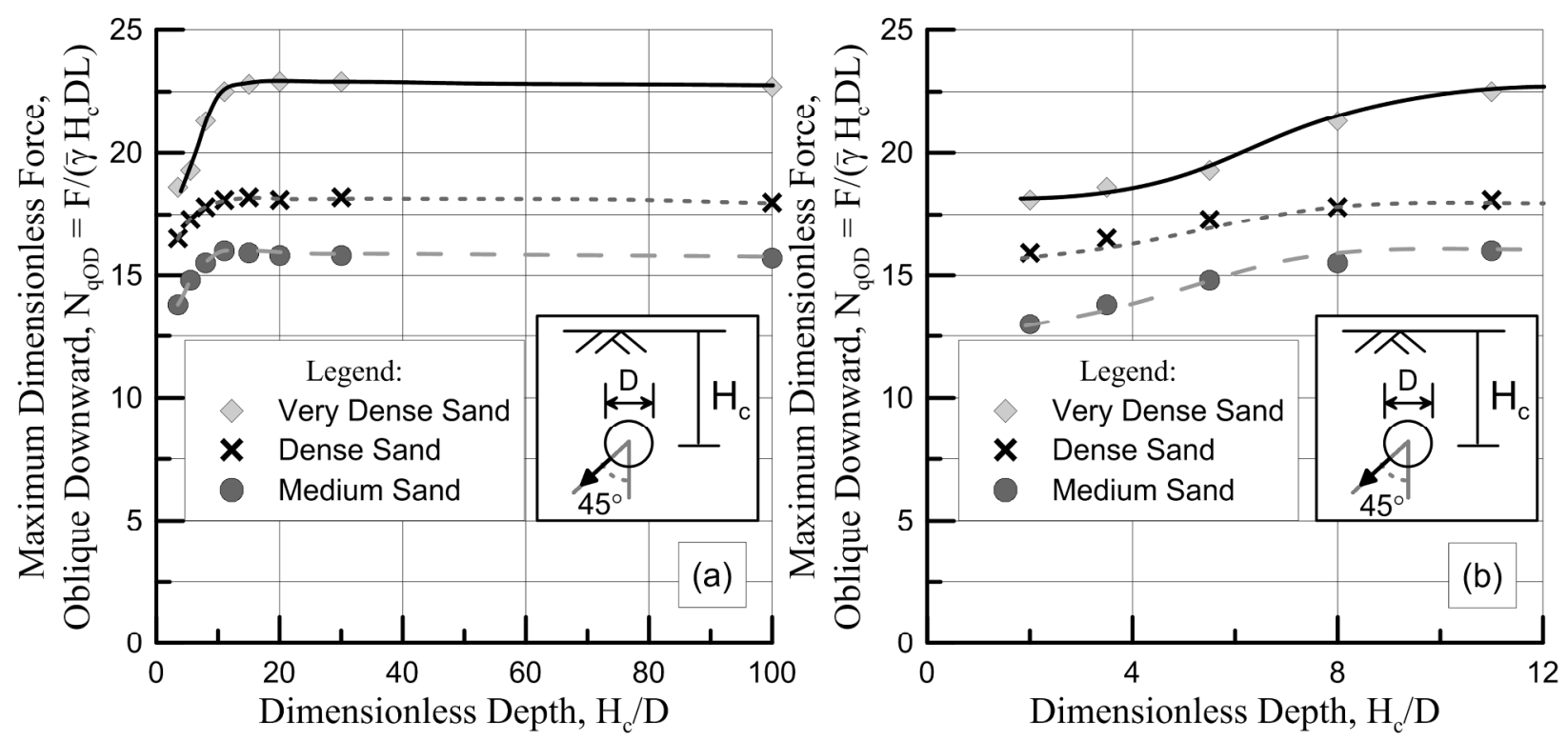

Figure 14. Dimensionless Maximum Oblique Downward Force as a Function of Dimensionless Depth for (a) $H_{c} / D \leq 100$, and (b) $H_{c} / D \leq 11$
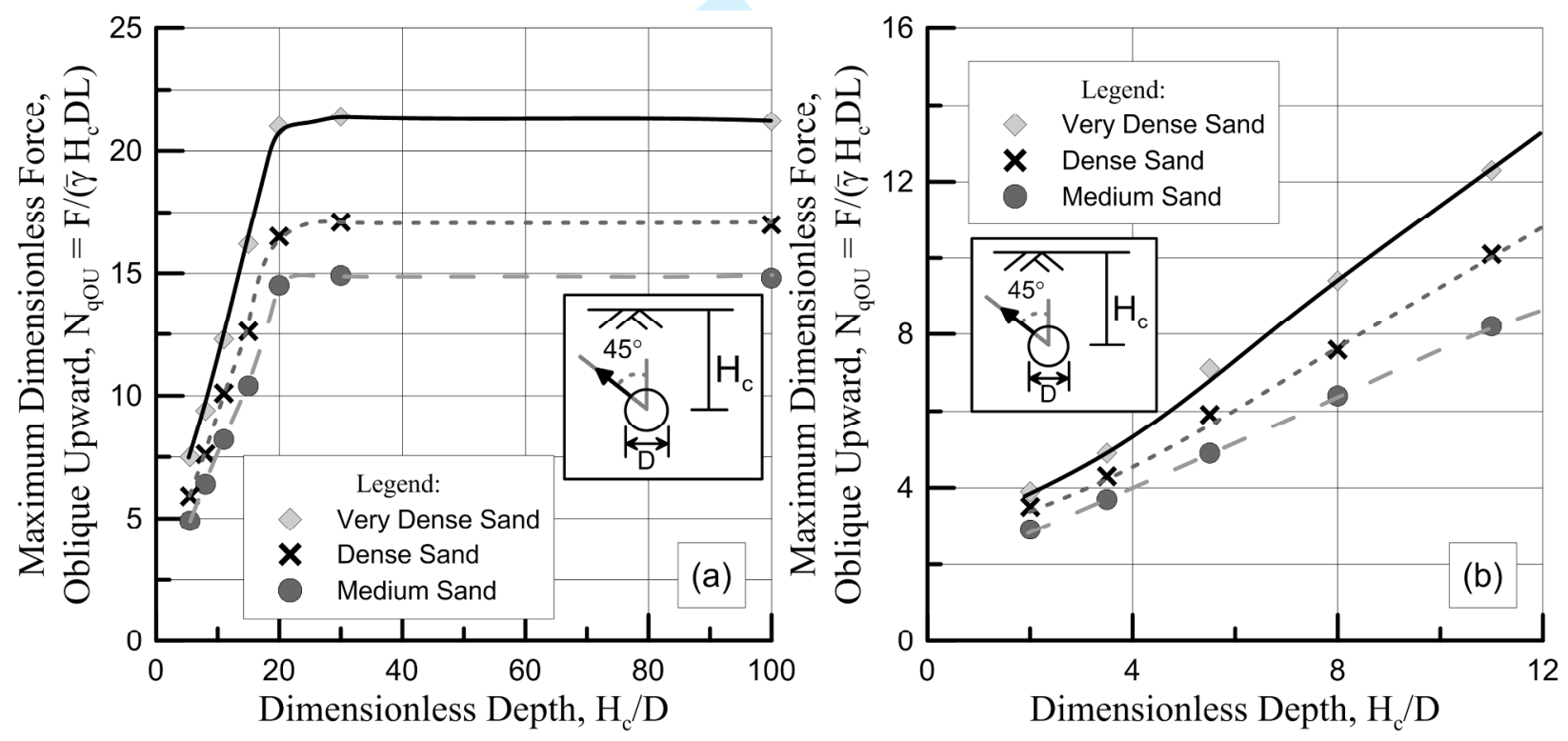

Figure 15. Dimensionless Maximum Oblique Upward Force as a Function of Dimensionless Depth for (a) $H_{c} / D \leq 100$, and (b) $H_{c} / D \leq 11$ 


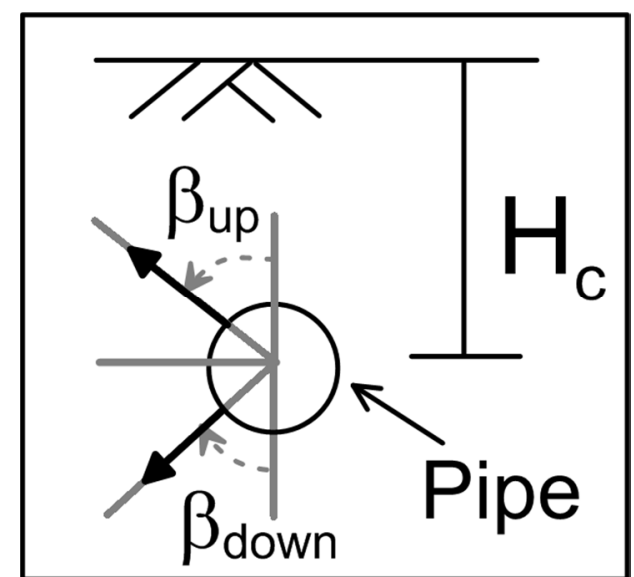

Figure 16. Angle Defining the Direction of Movement with Respect to Vertical at the Pipe Crown $\left(\beta_{\text {up }}\right)$ and at the Pipe Invert $\left(\beta_{\text {down }}\right)$ 


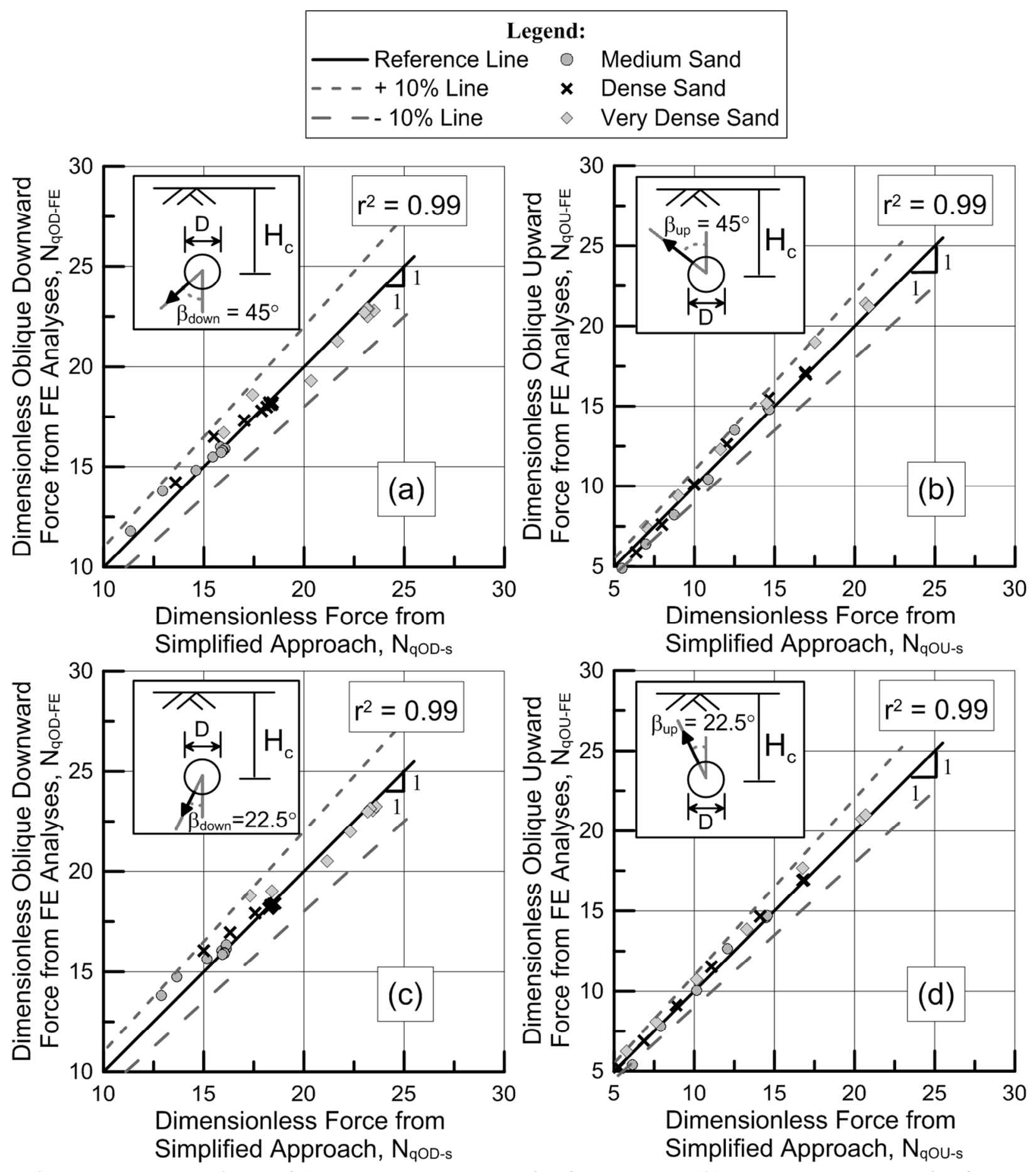

Figure 17. Comparison of (a) $\mathrm{N}_{\mathrm{qOD}-\mathrm{FE}} \mathrm{vs.} \mathrm{N}_{\mathrm{qOD}-\mathrm{s}}$ for $\beta_{\text {down }}=45^{\circ}$, (b) $\mathrm{N}_{\mathrm{qOU}-\mathrm{FE}} \mathrm{vs} . \mathrm{N}_{\mathrm{qOU}-\mathrm{s}}$ for $\beta_{\text {up }}=$ $45^{\circ}$, (c) $\mathrm{N}_{\mathrm{qOD}-\mathrm{FE}} \mathrm{Vs} . \mathrm{N}_{\mathrm{qOD}-\mathrm{s}}$ for $\beta_{\mathrm{down}}=22.5^{\circ}$, and (d) $\mathrm{N}_{\mathrm{qOU}-\mathrm{FE}} \mathrm{Vs} . \mathrm{N}_{\mathrm{qOU}-\mathrm{s}}$ for $\beta_{\text {up }}=22.5^{\circ}$ 


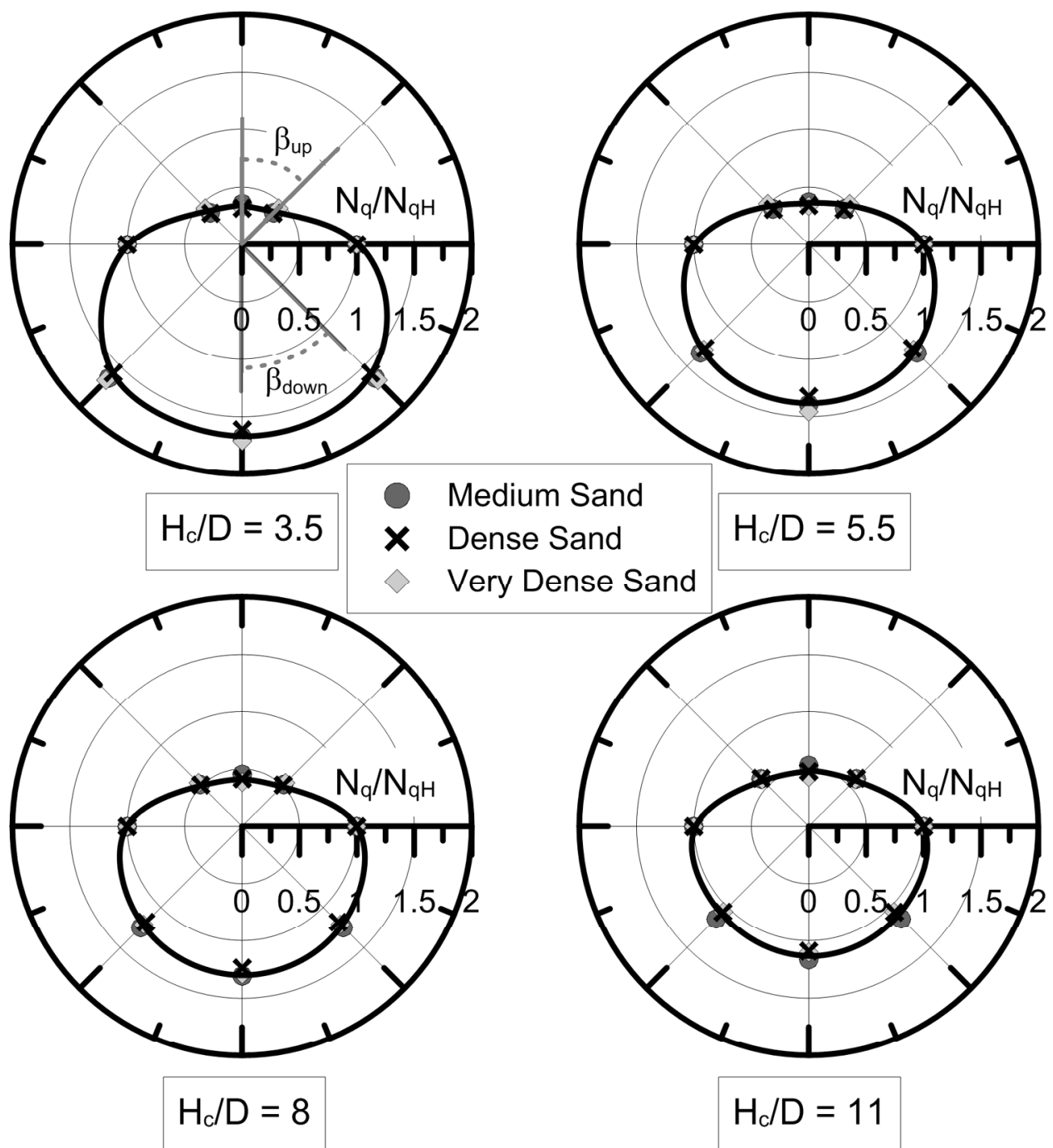

Figure 18. Visualization of $\mathrm{N}_{\mathrm{q}} / \mathrm{N}_{\mathrm{qH}}$ for $\mathrm{H}_{\mathrm{c}} / \mathrm{D}=3.5-11$ 


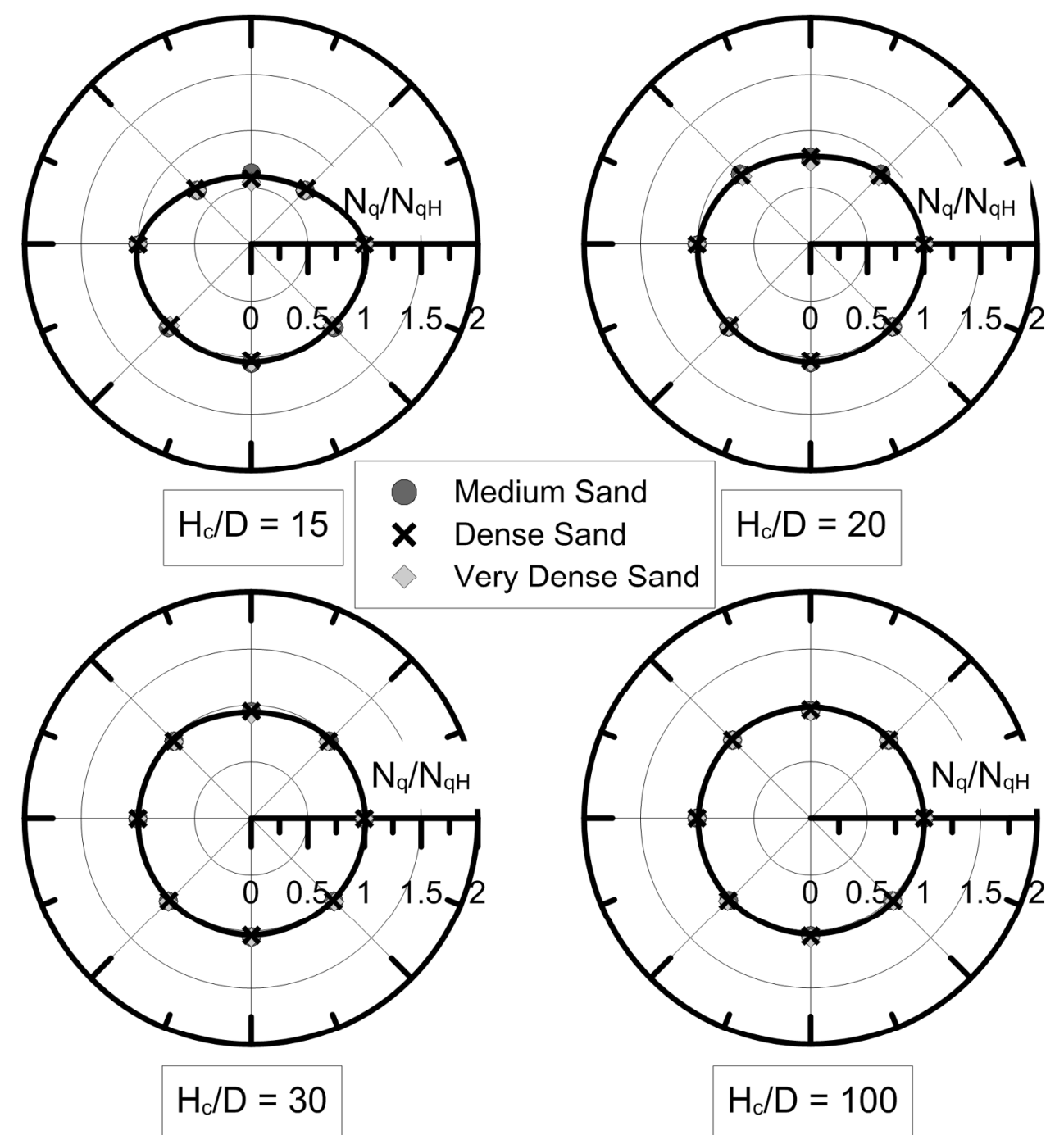

Figure 19. Visualization of $\mathrm{N}_{\mathrm{q}} / \mathrm{N}_{\mathrm{qH}}$ for $\mathrm{H}_{\mathrm{c}} / \mathrm{D}=15-100$ 

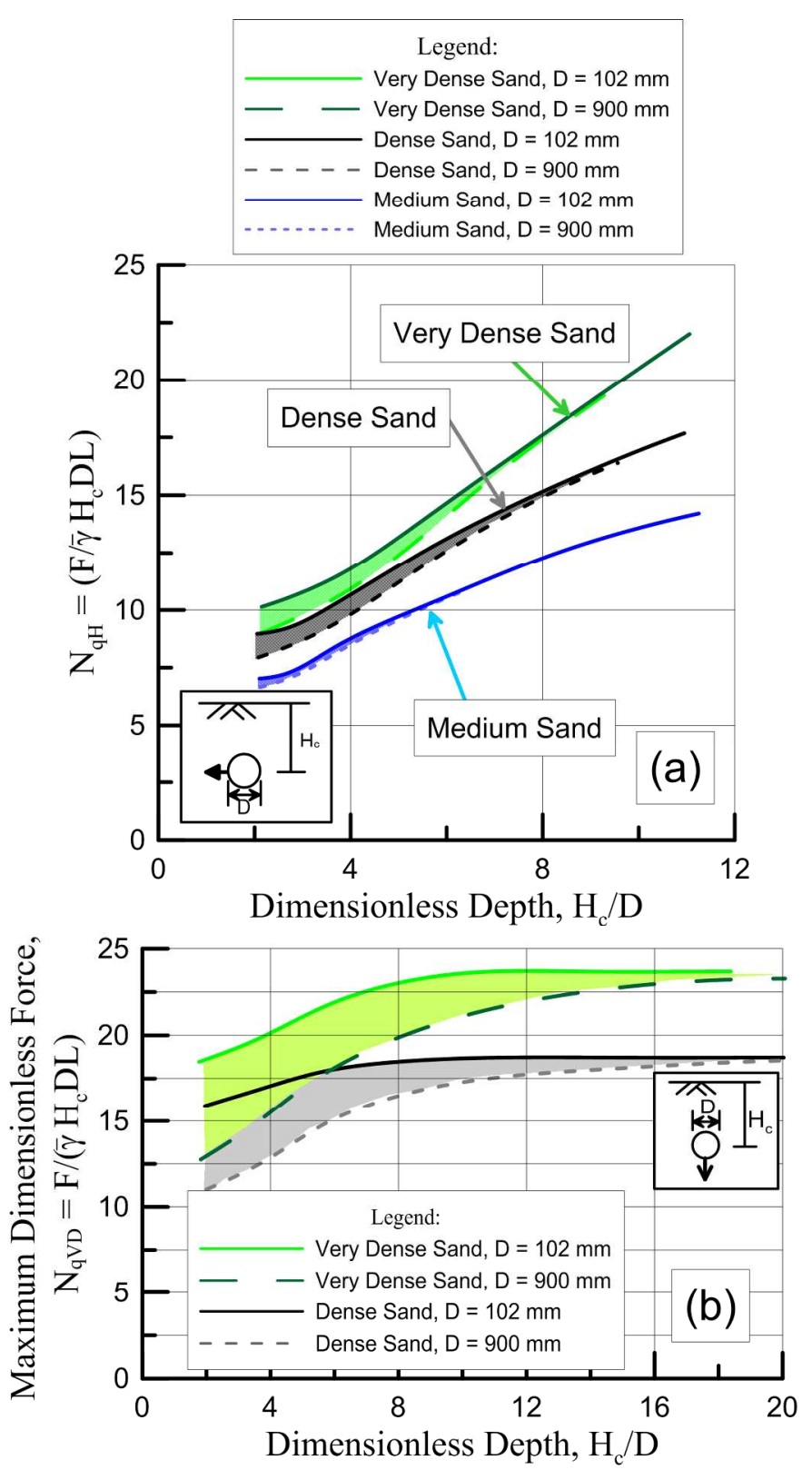

Figure 20. Plot of (a) $\mathrm{N}_{\mathrm{qH}}$ vs. $\mathrm{H}_{\mathrm{c}} / \mathrm{D}$ and (b) $\mathrm{N}_{\mathrm{qVD}}$ vs. $\mathrm{H}_{\mathrm{c}} / \mathrm{D}$ for Various Pipe Diameters 


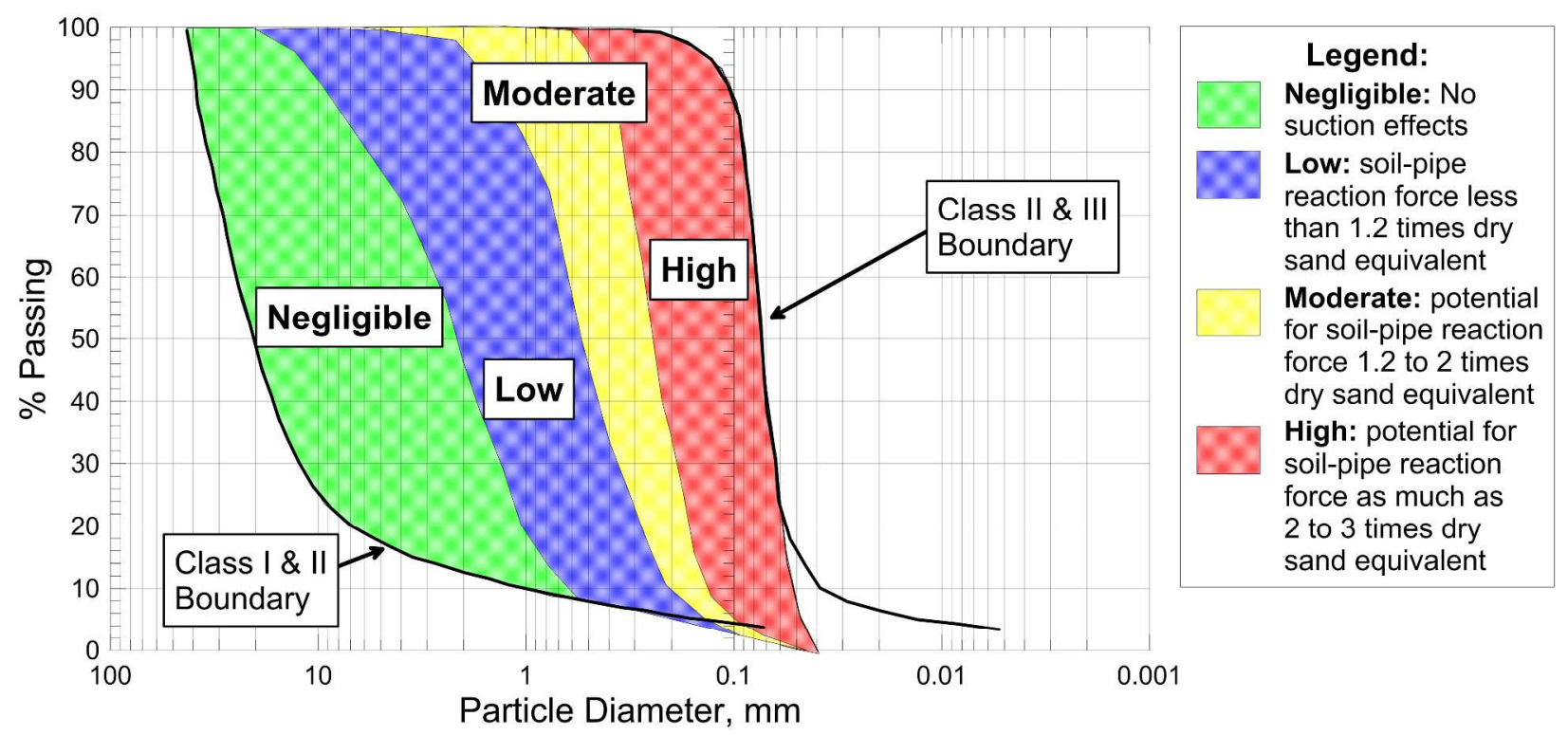

Figure 21. Suction Effects in Sand Related to Grain Size Distribution (after Robert et al. 2016) 

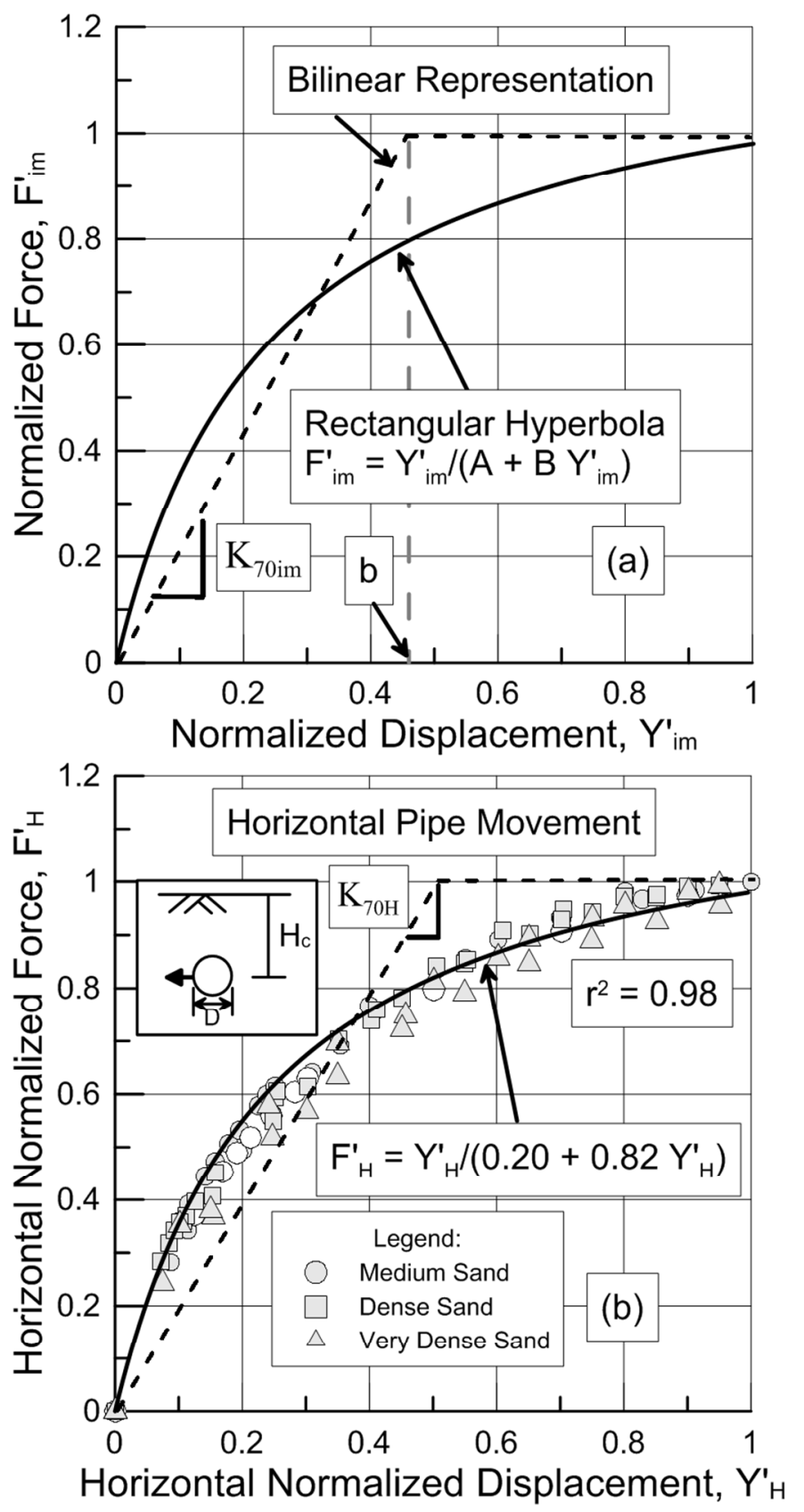

Figure 22. Hyperbolic and Bi-Linear Force vs. Displacement Relationship of (a) Generic Form, and (b) Horizontal Pipe Movement 


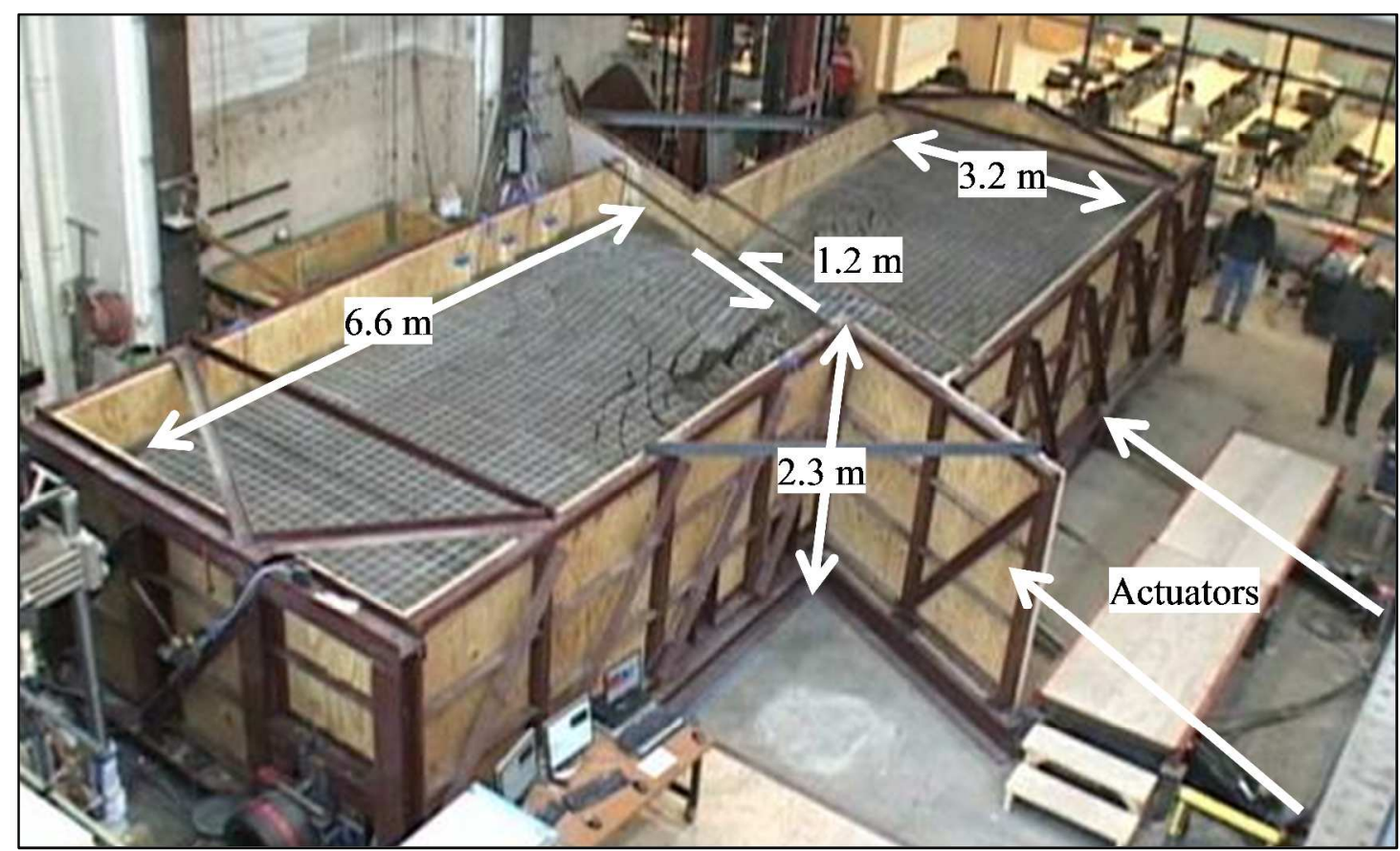

Figure 23. Large-Scale Split-Basin Test Facility at the Cornell University.
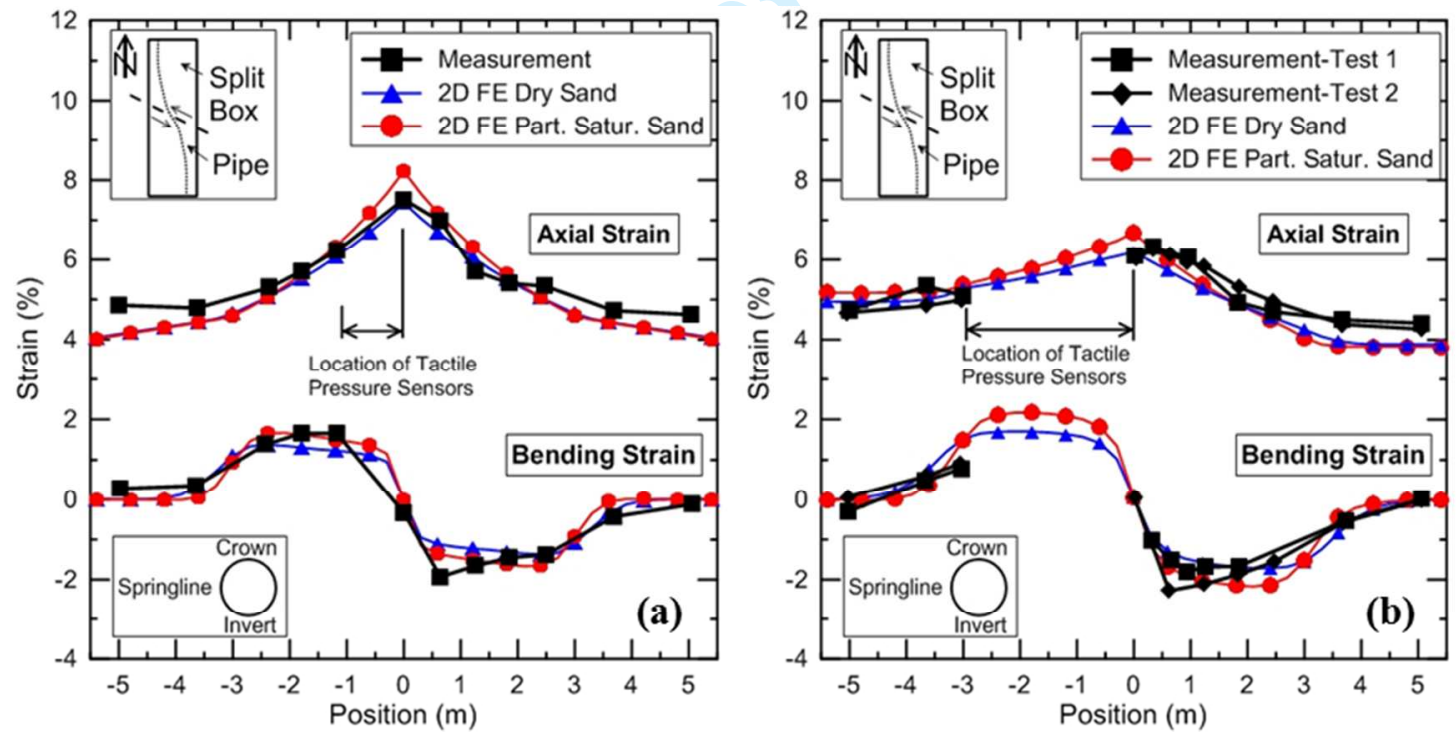

Figure 24. Measured and Numerically Simulated Strains for Large-Scale Test of Strike-Slip Faulting on a) 250-mm and b) 400-mm HDPE Pipelines 


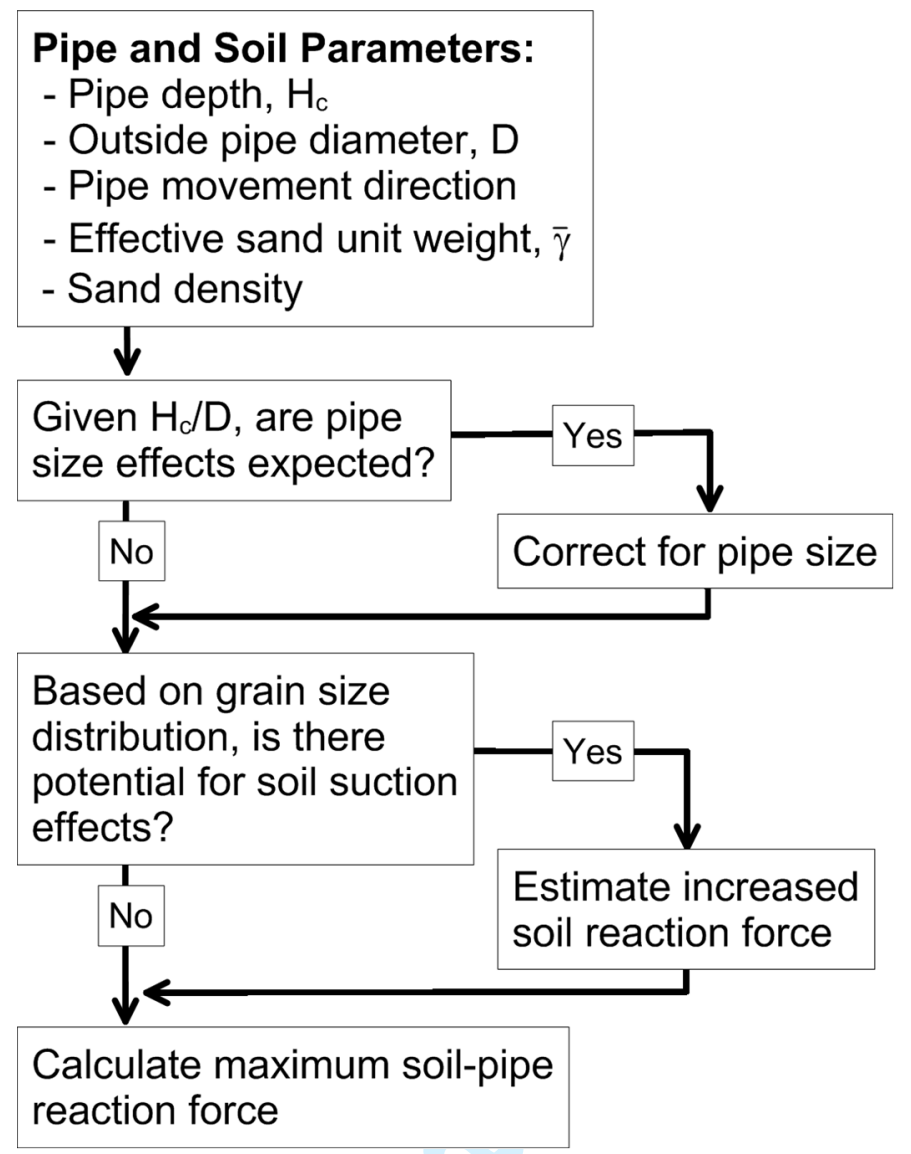

Figure 25. Flowchart for Estimation of Maximum Soil-Pipe Reaction Force 
Table 1. Summary of Representative Peak Angles of Shear Resistance and Angles of Dilatency for Medium, Dense, and Very Dense Sand

\begin{tabular}{|c|c|c|c|c|}
\hline Density & $\begin{array}{c}\gamma_{\mathrm{d}} \\
\left(\mathrm{kN} / \mathrm{m}^{3}\right)\end{array}$ & $\begin{array}{c}\phi^{\prime}{ }_{\text {ds-p }} \\
\text { (degrees) }\end{array}$ & $\begin{array}{c}\phi_{\text {ps-p }}^{\prime} \\
(\text { degrees })\end{array}$ & $\begin{array}{c}\psi_{\mathrm{p}} \\
(\text { degrees })\end{array}$ \\
\hline Medium & $15.5 \sim 16.5$ & $35 \sim 37$ & $41 \sim 44$ & $3 \sim 6$ \\
\hline Dense & $16.5 \sim 17.5$ & $38 \sim 40$ & $45 \sim 47$ & $7 \sim 10$ \\
\hline Very Dense & $17.5 \sim 18.0$ & $41 \sim 43$ & $48 \sim 49$ & $11 \sim 17$ \\
\hline
\end{tabular}

Table 2. Summary of Dry and Partially Saturated Sand Strength Parameters

\begin{tabular}{|c|c|c|c|c|c|c|c|}
\hline $\begin{array}{c}\text { Density } \\
\text { Description }\end{array}$ & Soil & $\begin{array}{c}\gamma_{\mathrm{d}} \\
\left(\mathrm{kN} / \mathrm{m}^{3}\right)\end{array}$ & $\begin{array}{c}\phi_{\text {ps-p }}^{\prime} \\
(\mathrm{degrees})\end{array}$ & $\begin{array}{c}\psi_{\mathrm{p}} \\
(\mathrm{degrees})\end{array}$ & $\begin{array}{c}\gamma_{\text {total }} \\
\left(\mathrm{kN} / \mathrm{m}^{3}\right)\end{array}$ & $\begin{array}{c}\phi_{\mathrm{ps}-\mathrm{p}} \\
(\mathrm{degrees})\end{array}$ & Full-scale Test Results \\
\hline Medium & $\begin{array}{c}\text { Dry CU } \\
\text { Filter Sand }\end{array}$ & 16.4 & 43.0 & 5.7 & N/A & N/A & $\begin{array}{c}\text { Trautmann and } \\
\text { O'Rourke (1985) }\end{array}$ \\
\hline Dense & $\begin{array}{c}\text { Dry RMS } \\
\text { Graded Sand }\end{array}$ & 17.1 & 46.3 & 7.5 & N/A & N/A & $\begin{array}{c}\text { Olson (2009), } \\
\text { Turner (2004) }\end{array}$ \\
\hline Very Dense & $\begin{array}{c}\text { Dry CU } \\
\text { Filter Sand }\end{array}$ & 17.7 & 48.9 & 14.2 & N/A & N/A & $\begin{array}{c}\text { Trautmann and } \\
\text { O'Rourke (1985) }\end{array}$ \\
\hline N/A & $\begin{array}{c}\text { Partially Saturated } \\
\text { RMS Graded Sand }\end{array}$ & 16.0 & N/A & 10.8 & 16.6 & 43.7 & Olson (2009) \\
\hline
\end{tabular}

Table 3. Summary of Hyperbolic and Bi-Linear Parameters for Force vs. Displacement Relationships for Dry Sand

\begin{tabular}{|c|c|c|c|c|c|c|}
\hline \multirow{2}{*}{$\begin{array}{l}\text { Interaction } \\
\text { Mode (im) }\end{array}$} & \multicolumn{3}{|c|}{ Hyperbolic Constants } & Bi-Linear & \multirow{2}{*}{$\begin{array}{l}\text { Sand } \\
\text { Density }\end{array}$} & \multirow{2}{*}{$Y_{\operatorname{mim}}$} \\
\hline & $\mathrm{A}$ & $\mathrm{B}$ & $\mathrm{r}^{2}$ & $\mathrm{~b}^{*}$ & & \\
\hline \multirow{3}{*}{$\begin{array}{l}\text { Vertical } \\
\text { Upward (VU) }\end{array}$} & \multirow{3}{*}{0.1} & \multirow{3}{*}{0.88} & \multirow{3}{*}{0.96} & \multirow{3}{*}{0.3} & Medium & $0.013 \mathrm{H}_{\mathrm{c}}{ }^{* *}$ \\
\hline & & & & & Dense & $0.011 \mathrm{H}_{\mathrm{c}}$ \\
\hline & & & & & Very Dense & $0.010 \mathrm{H}_{\mathrm{c}}$ \\
\hline \multirow{3}{*}{$\begin{array}{l}\text { Oblique } \\
\text { Upward (OU) }\end{array}$} & \multirow{3}{*}{0.19} & \multirow{3}{*}{0.83} & \multirow{3}{*}{0.98} & \multirow{3}{*}{0.45} & Medium & $0.017 \mathrm{H}_{\mathrm{c}}$ \\
\hline & & & & & Dense & $0.013 \mathrm{H}_{\mathrm{c}}$ \\
\hline & & & & & Very Dense & $0.012 \mathrm{H}_{\mathrm{c}}$ \\
\hline \multirow{3}{*}{$\begin{array}{l}\text { Horizontal } \\
\text { (H) }\end{array}$} & \multirow{3}{*}{0.20} & \multirow{3}{*}{0.82} & \multirow{3}{*}{0.98} & \multirow{3}{*}{0.5} & Medium & $0.037 \mathrm{H}_{\mathrm{c}}$ \\
\hline & & & & & Dense & $0.027 \mathrm{H}_{\mathrm{c}}$ \\
\hline & & & & & Very Dense & $0.021 \mathrm{H}_{\mathrm{c}}$ \\
\hline \multirow{3}{*}{$\begin{array}{l}\text { Oblique } \\
\text { Downward } \\
\text { (OD) }\end{array}$} & \multirow{3}{*}{0.45} & \multirow{3}{*}{0.55} & \multirow{3}{*}{0.99} & \multirow{3}{*}{0.75} & Medium & $0.010 \mathrm{H}_{\mathrm{c}}$ \\
\hline & & & & & Dense & $0.009 \mathrm{H}_{\mathrm{c}}$ \\
\hline & & & & & Very Dense & $0.008 \mathrm{H}_{\mathrm{c}}$ \\
\hline \multirow{3}{*}{$\begin{array}{l}\text { Vertical } \\
\text { Downward } \\
\text { (VD) }\end{array}$} & \multirow{3}{*}{0.53} & \multirow{3}{*}{0.46} & \multirow{3}{*}{0.99} & \multirow{3}{*}{0.8} & Medium & $0.008 \mathrm{H}_{\mathrm{c}}$ \\
\hline & & & & & Dense & $0.008 \mathrm{H}_{\mathrm{c}}$ \\
\hline & & & & & Very Dense & $0.007 \mathrm{H}_{\mathrm{c}}$ \\
\hline
\end{tabular}

$*-b=$ normalized displacement at the maximum force

** $-\mathrm{H}_{\mathrm{c}}=$ depth to the center of pipe 